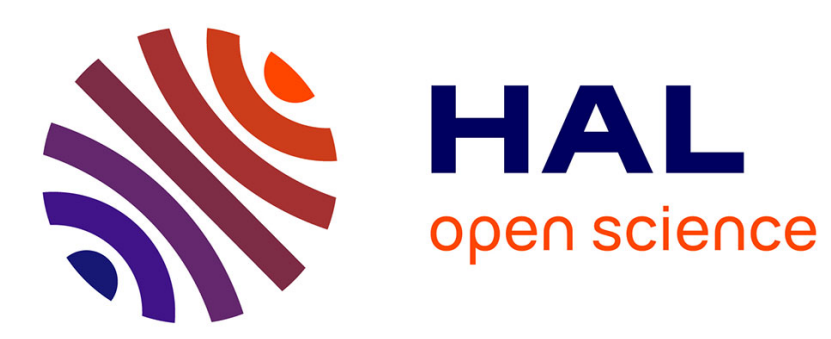

\title{
Speckle noise spectrum at near-nadir incidence angles for a time-varying sea surface
} Ping Chen, Danièle Hauser, Shihao Zou, Jianyang Si, Eva Le Merle

\section{To cite this version:}

Ping Chen, Danièle Hauser, Shihao Zou, Jianyang Si, Eva Le Merle. Speckle noise spectrum at nearnadir incidence angles for a time-varying sea surface. IEEE Transactions on Geoscience and Remote Sensing, 2022, 60, pp.4200320. 10.1109/TGRS.2020.3037910 . insu-03008365

\section{HAL Id: insu-03008365 https://hal-insu.archives-ouvertes.fr/insu-03008365}

Submitted on 16 Nov 2020

HAL is a multi-disciplinary open access archive for the deposit and dissemination of scientific research documents, whether they are published or not. The documents may come from teaching and research institutions in France or abroad, or from public or private research centers.
L'archive ouverte pluridisciplinaire HAL, est destinée au dépôt et à la diffusion de documents scientifiques de niveau recherche, publiés ou non, émanant des établissements d'enseignement et de recherche français ou étrangers, des laboratoires publics ou privés. 


\title{
Speckle noise spectrum at near-nadir incidence angles for a time-varying sea surface
}

\author{
Ping Chen, Danièle Hauser, IEEE Member, Shihao Zou, Jianyang Si, Eva Le Merle
}

\begin{abstract}
Speckle noise is inherent to radar measurements. For applications which need both a high temporal and high spatial resolution, a classical method for the reduction of the speckle noise by filtering the backscattered signal may not be sufficient. In particular, when radar observations are used to estimate ocean wave spectra from relative fluctuations of the radar signal within a given footprint, a method must be implemented to correct for the speckle effect in the Fourier domain (i.e. density spectrum). A theoretical background to model the speckle density spectrum for a radar with near-nadir incidences was proposed by Jackson in 1981 but it is based on a stationary sea surface assumption and ignores the variation of the main factor in the four-frequency moment near the origin. In this paper, we revisit this theoretical background to extend this model to a time-varying sea surface and alleviate some assumptions on the Fresnel phase formulation. The results from the model applied in the configuration of an airborne system indicate that not only the displacement of the radar but also the dynamic properties of the sea surfaces have a significant effect on the speckle noise spectrum in certain directions of observations. The effects depend on the radar look direction in azimuth, and on sea surface conditions (wind speed, wind direction with respect to the aircraft route, surface wave spectrum). This new model is validated against observations of the airborne near-nadir incidence scatterometer-KuROS. We show in particular that the errors between the experimental estimation of the omni-directional speckle noise spectrum from KuROS and the prediction by our model are below $10 \%$.
\end{abstract}

Index Terms - speckle noise spectrum, time-varying sea surface, near-nadir incidence, Geometry Optical approximation

\section{INTRODUCTION}

Speckle noise is inherent to radar measurements used to sense geophysical media composed of distributed scattering elements (e. g., sea surface). Indeed, the composite backscatter generated by these media is the coherent sum of components backscattered from individual elements which generate different phases and amplitudes, and add together to give a resultant whose intensity varies randomly. Thus, the backscattered signal measured by a radar exhibits fluctuations from one look to the next one, even when the surface is the same. When radar systems are used to estimate ocean wave properties from the analysis of signal fluctuations at a relatively high spatial resolution (typically 5 to $30 \mathrm{~m}$ horizontally), the speckle noise is a perturbing effect which must be accounted for in the inversion of the fluctuation spectra into wave spectra [1], [2], [3], [4].

There are presently two main categories of radar systems

Manuscript received September 04, 2020; accepted October 26, 2020. This work was supported in part by the National Key Research and Development Program of China (2016YFC1401005), the National Natural Science Foundation of China under Grant 41976168, Grant 41976173, and Shandong Provincial Natural Science Foundation of China under Grant ZR2019MD016. (Corresponding author: Ping Chen.)

Ping Chen, Shihao Zou, and Jianyang Si are with the School of Electronic Information and Communications, Huazhong University of Science and Technology, Wuhan, 430074, China (e-mail:chenping@hust.edu.cn; zoushshawn@163.com; m201972058@hust.edu.cn).

Danièle Hauser, and Eva Le Merle are with the LATMOS, Université Paris-Saclay, UVSQ, CNRS, Sorbonne Université, 78280 Guyancourt,

France(email:daniele.hauser@latmos.ipsl.fr ;eva.lemerle@latmos.ipsl.fr) devoted to the measurement of ocean wave spectra: those based on a SAR side-looking configuration (see e.g. [2], [5], [6], [7], [8]), and those based on a RAR real-aperture and azimuthally scanning configuration [9], [10]. In both cases the spectrum of ocean waves is derived from the spectra of signal fluctuations analyzed over a footprint of several kilometers. In both cases, it requires to subtract the speckle noise spectrum from the fluctuation spectrum.

Jackson provided the theoretical background to model the speckle noise contribution in the Fourier domain (spectrum of signal fluctuations in space and time) for a real-aperture nearnadir looking radar [1]. Based on this theoretical approach, a simple formula was then proposed to represent the speckle density spectrum as a function of the wave number at the surface [11]. Later, Hauser proposed an empirical method to derive the parameters of the Jackson's model speckle spectrum from airborne observations with a scanning real-aperture radar [3]. It consists in estimating a mean spectrum of speckle from a combination of fluctuation spectra obtained with radar signals integrated over different durations. This speckle spectrum is then subtracted from the fluctuation spectrum to estimate the modulation spectrum due to waves.

Engen and Johnsen proposed another method the so-called "cross-spectrum" method to remove the speckle noise contribution from SAR observations over the ocean using successive looks [2]. It is based on the conjugate product of successive fluctuation spectra obtained from overlapping scenes. This method is now operationally used by The European Space Agency to provide wave spectra from SAR images. It was also later used by Caudal [10] and Le Merle [12] in the processing of their airborne real-aperture scanning radar observations to retrieve wave spectra.

Both empirical methods (based on cross-spectra or on multiduration time integration) are very efficient but are constrained by the conditions of measurements (appropriate overlap of successive looks). With the recent launch of the CFOSAT satellite and its real-aperture wave spectrometer SWIM in operation, the question of speckle estimation is still of actuality, because the SWIM mode of operation is not well appropriate neither to the cross-spectral method (limited overlap of successive scenes in the nominal mode of acquisition) nor to the multi-integration method (loss of range resolution in this case). Therefore, an empirical ad'hoc speckle model was estimated from the SWIM observations [4] and is currently used to invert the wave spectra from the fluctuation spectra. The results indicate that this empirical speckle spectrum shows an important increase in a sector of $\pm 15^{\circ}$ and that its level depends on azimuth with respect to the flight-track, on latitude, and on sea-surface conditions.

Although all the proposed empirical methods to estimate or subtract the speckle noise spectrum are convenient from an operational point of view, they are still difficult to reconcile with the existing theoretical background. Therefore, in the present study we propose to revisit the analytical model of Jackson of speckle noise spectrum.

Jackson established an analytical speckle noise spectrum model based on the Geometry Optical approximation for the 
scattering processes expressed for a frozen sea surface [1], a pulse-limited transmitted waveform with a given range resolution and integration time. This model takes into account the displacement of the illuminated ocean scene with respect to the radar in an azimuthal scanning geometry, but it does not take into account the surface scatter motion. The consequence is that, according to this model, the speckle noise spectrum along the flight direction tends to very large values because the number of independent samples forming the integrated radar echo is supposed to tend to one. However, this is in contradiction with the actual situation as seen from both airborne and spaceborne observations, which indicate that in spite of an important increase of speckle when the radar look direction is approximatively aligned with the platform displacement the number of independent samples deduced from the speckle noise spectrum is far more than 1 .

We will demonstrate in this paper that this is because in certain conditions, the kinematic properties of the sea surface cannot be ignored. Indeed, during the radar integration time (several tens micro-seconds typically), the correlation time of the scattered field from the sea surface cannot be ignored, since it is typically a few milliseconds [13].

Jackson proposed a simplified analytical expression of his model of speckle spectrum under the assumption of a Gaussian shaped transmitted wave form [11]. However, because the dynamic properties of the sea surface were ignored, the speckle noise spectrum density along the flight direction estimated from this modified model is still much larger than the one measured. Up to now there is no analytical model of speckle noise spectrum in which both the radar motion and timevarying properties of the sea surface are taken into account.

This is precisely the aim of this paper to propose an analytical noise spectrum model valid for a radar with nearnadir incidences, and a time-varying sea surface. There are two differences compared with Jackson's initial publication [1]. The first is that in addition to the movement of the radar, we take into account the kinematic properties of sea surface during the radar integration time. The second is that in our approach, we remove the assumption used in Jackson's model that the variation of the main factor in the four-frequency moment of the surface scattering matrix near the origin tends to zero. Furthermore, we propose here a validation of the model by using independent speckle spectrum estimates from observations.

In Section II, the speckle spectrum model for a time-varying sea surface is presented with the movements of both radar and sea surface scatters taken into account. In Section III, we present results obtained with this model and a prescribed sea state (imposing a surface wave height and wind) for different sea-surface conditions and for a radar configuration corresponding to airborne observations. In section IV, we present empirical estimates of the speckle noise spectrum obtained from the airborne real-aperture azimuthally scanning KuROS radar [4]. In section $\mathrm{V}$, we compare the speckle spectra predicted by the model to those estimated directly from the KuROS observations. In section VI, we extend our analysis of the model results to other configurations of observations (other radar frequency, platform speed, incidence angles, footprint dimension). Conclusion and perspectives are drawn in section VII.

\section{MODEL OF SPECKLE NOISE SPECTRUM FOR AN OCEAN WAVE SCATTEROMETER OVER A MOVING SURFACE}

Jackson expressed the ensemble average fluctuation spectrum of the signal as a function of frequency $\omega$ as:

$$
\begin{aligned}
\widetilde{\mathrm{P}}(\omega)=\iiint W\left(\Omega_{0}-\vec{K} \cdot \vec{V}\right) \mathrm{N}\left(v, v^{\prime}, \omega, \Omega_{0}\right) E_{0}(v) \cdot \\
E_{0}^{*}(v-\omega) \cdot E_{0}^{*}\left(v^{\prime}\right) \cdot E_{0}\left(v^{\prime}-\omega\right) d \Omega_{0} d v d
\end{aligned}
$$

where $E_{0}(v)$ is the Fourier Transform (FT) of the incident short-pulsed waveform, $W$ is the filter window associated to the integration of the signal over an integral time $T_{\text {int }}$, $N\left(v, v^{\prime}, \omega, \Omega_{0}\right)$ is the FT of the 'four-frequency' moment of the surface scattering function (see below), $\vec{K} \cdot \vec{V}$ is the Doppler frequency induced by the platform motion $\vec{V}, \vec{K}$ the wavenumber at the surface, and the asterisk denotes complex conjugate. Expressions of these terms are given in [1] and recalled below:

$$
\begin{aligned}
& W\left(\Omega_{0}\right)=\left[\frac{\sin \left(\Omega_{0} \cdot \frac{T_{\text {int }}}{2}\right)}{\Omega_{0} \cdot \frac{T_{\text {int }}}{2}}\right]^{2} \\
& N\left(v, v^{\prime}, \omega, \Omega_{0}\right)=\int \frac{1}{2 \pi} M\left(v, v^{\prime}, \omega, \Delta t\right) \exp \left(i \Omega_{0} \Delta t\right) d \Delta t
\end{aligned}
$$

$$
\begin{aligned}
M\left(v, v^{\prime}, \omega, \Delta t\right)=< & S(v, t) \cdot S^{*}(v-\omega, t) \cdot S^{*}\left(v^{\prime}, t+\Delta t\right) \\
& \cdot S\left(v^{\prime}-\omega, t+\Delta t\right)>
\end{aligned}
$$

$M\left(v, v^{\prime}, \omega, \Delta t\right)$ is a four-frequency moment defined from the surface scattering transfer function $S(v, t)$, where $t$ is the 'slow time' in the radar chronogram, $v, v^{\prime}$ are the angular frequency of the electromagnetic wave, $\omega$ is the angular frequency difference, $\Delta t$ is the time interval (the full list of symbols is given in Appendix B).

Equation (1-4) here above are identical to (7-12) in [1]. Jackson then transposes this formalism to the wavenumber domain by writing, $v=k c, v^{\prime}=k^{\prime} c, \omega=\kappa c, \Delta v=v-$ $v^{\prime}, \Delta k=k-k^{\prime}, \vec{K}=2 \kappa \sin \theta \vec{\rho}, \Delta K=2 \Delta k \sin \theta$, where $c$ is the light speed, $\vec{\rho}$ is the unit vector of the range direction of the radar, and $\theta$ the incidence angle.

Then, $S(v, t)$ is developed using the geometrical optics scattering approximation which is valid at near-nadir incidence angles [14]. Assuming a Gaussian shape of the transmitted pulse and a Gaussian pattern for the antenna beam, Jackson finally obtains the spectrum of the signal (integrated over $T_{\text {int }}$ ) in wavenumber as the sum of two terms [1], [11], one associated with fluctuations due to the long detected waves (through their tilting impact on the backscattered signal) and the other one with the fading (speckle) noise.

Considering a more realistic shape of the transmitted pulse, namely a sinus cardinal function $e_{0}(x)=\operatorname{sinc}\left(\frac{x}{\delta x}\right)$, with $\delta x=\frac{c}{2 B \sin \theta}$, where B is the bandwidth of the transmitted pulse, and $\operatorname{sinc}(x)=\frac{\sin (\pi x)}{\pi x}$, with $x$ is the horizontal range along the range direction $\vec{\rho}$ of the radar, then the speckle noise spectrum of Jackson's model when applied to the relative fluctuations of the backscattering coefficient becomes:

$\mathrm{P}_{s p_{-} J a c}(K, \Phi)=\operatorname{tri}\left(\frac{K}{2 \pi K_{p}}\right) \frac{1}{2 \pi K_{p} N_{J a c}(\Phi)}$

where tri is the triangle function, $K$ is the wavenumber at the surface, $\Phi$ is the azimuth angle relative to the flight direction,

$K_{p}=\frac{1}{\delta x}=\frac{2 B \sin \theta}{c}$

$N_{\text {Jac }}$ is the number of independent samples due to the radar displacement:

$N_{\text {Jac }}(\Phi)=T_{\text {int }} \frac{2 V}{\lambda} \beta_{\phi} \sin \Phi$

where $\beta_{\phi}$ is the one-way antenna aperture in azimuth, $V$ the platform speed and $\lambda$ the radar wavelength.

In the derivation of (5), Jackson used two assumptions. 
Firstly, the sea surface is assumed frozen over the $T_{\text {int }}$ duration: the sea surface height of a point $\vec{x}$ on the surface is expressed as $\xi(\vec{x})$. This is a strong assumption. Indeed, for ocean wave scatterometer, the value of $T_{\text {int }}$ is the order of ten microseconds, typically 30 to $40 \mathrm{~ms}$ for SWIM [4], 33ms for KuROS [10]. However, at $\mathrm{Ku}$ band, the correlation time of the electromagnetic field scattered by the sea surface is expected to be of the order of millisecond [13]. Thus, the dynamic properties of the sea surface should be taken into account during the $T_{\text {int }}$ duration. Especially, when the observation azimuth angle is close to the flight direction, the number of independent samples induced by the movement of the platform $\left(N_{J a c}\right)$ is close to one, so that the number of actual independent samples is mainly determined by the movement of the sea surface scatters. Thus, for radar observations obtained with an azimuthally scanning system, besides the movement of the radar, the instantaneous velocity of the sea surface scatters must be included in a model of speckle noise spectrum. The second hypothesis by Jackson is that the variation of the main factor in the four-frequency moment near the origin tends to 0 [1]. This assumption simplifies the derivation greatly, but its validity needs to be assessed.

Note also that Jackson, added a coefficient $(1+\mu)$ in (5) [11], to account for the variation of speckle noise on the signal modulated by the tilting waves:

$\mathrm{P}_{s p_{-} J a c}^{\prime}(K, \Phi)=\operatorname{tri}\left(\frac{K}{2 \pi K_{p}}\right) \frac{1}{2 \pi K_{p} N^{\prime} J a c}(\Phi)$

$N^{\prime}{ }_{J a c}(\Phi)=N_{J a c}(\Phi) /(1+\mu)$

$\mu=\int P_{\text {mod }}(K, \Phi) d K$

where $P_{\text {mod }}$ is the spectrum of the relative fluctuations due to the presence of long waves.

However, the effect of $P_{\text {mod }}$ on the speckle is accounted for in our model by another way, as explained below and in the appendix.

In order to extend Jackson's speckle noise spectrum theory to a time-varying sea surface, we expressed the surface elevation as a time and space variable $\xi(\vec{x}, t)$. Substituting $\xi(\vec{x}, t)$ into the surface scattering transfer function $S(v, t)$, and assuming the sea surface to be stationary in the mean and ergodic, then applying Longuest-Higgins' method [15] to expand the bracket term $\langle\cdots\rangle$ in four-frequency moment $M\left(v, v^{\prime}, \omega, \Delta t\right)$ and calculating four integrations about all $\overrightarrow{x_{l}}$, $\mathrm{i}=1,2,3,4$, the fluctuation spectrum equation for a time-varying sea surface is obtained (See Appendix A for details). Accordingly, the new speckle noise spectrum model is

$\mathrm{P}_{s p}(K, \Phi)=\operatorname{tri}\left(\frac{K}{2 \pi K_{p}}\right) \frac{1}{2 \pi K_{p} N_{\text {tot }}(\Phi)}$

Where $\frac{1}{N_{\text {tot }}}=\frac{1}{N_{\text {mov }}}+\frac{1}{N_{\text {int }}}$,

$N_{\text {mov }}(\Phi)=\sqrt{N_{\text {platf }}^{2}+N_{\text {surf }}^{2}}$,

$N_{\text {platf }}(\Phi)=N_{J a c}(\Phi)$,

$N_{\text {surf }}=\frac{2}{\sqrt{\pi}} T_{\text {int }} k \cos \theta \sqrt{m_{t t}}$

$\frac{1}{N_{\text {int }}}=\frac{\sqrt{\frac{\pi}{\hat{\alpha}}} \int P_{\text {mod }}^{*}(K, \Phi) d K}{T_{\text {int }}}$

where $\hat{\alpha}$ is a factor proportional to the surface velocity variance $m_{t t}$ :

$\hat{\alpha}=4 k^{2} \cos ^{2} \theta m_{t t}$,

and

$P_{\text {mod }}^{*}(K, \Phi)=P_{\text {mod }}(K, \Phi)+\frac{\sqrt{2 \pi}}{L_{\phi}} \frac{g}{2 m_{t t}} K^{2} F(K, \Phi)$

$m_{t t}=\int_{0}^{\omega_{d}} d \omega \omega^{2} F(\omega)$

$\omega_{d}$ in (8i) corresponds to the wave scale limit which makes the quasi-specular scattering approximation valid, generally, $1 / 5 \sim 1 / 3$ times radar wave number [3], [16]. The detailed method to determine $\omega_{d}$ will be described in section III. $F(K, \Phi)$ is the wave height directional spectrum, $F(\omega)$ is the omni-directional spectrum with the angular frequency $\omega, g$ is the acceleration of gravity. In (8b), the total number $N_{\text {tot }}$ of independent samples which govern the speckle noise spectrum is not only dependent on $N_{m o v}$, related to the movement between the radar and the sea surface, but also to $N_{\text {int }}$, whose expression involves integral quantities of the wave-spectrum. Compared with the original model of Jackson recalled in (6), our model combines in (8c) the contribution from $N_{\text {platf }}$ determined by the movement of the platform, and a contribution from the surface, $N_{\text {surf }}$, which is related to the sea surface velocity variances $m_{t t}$. Secondly, compared to (6), (8) has an additional term involving $N_{i n t}$, which results from the fact that the variation of the main factors in the four-frequency moment near the origin are no longer neglected. We will see in section IV that the integral term in $N_{\text {int }}$ is not negligible for some cases.

If ignoring the dynamic properties of the sea surfaces $\left(N_{\text {surf }}=0\right)$ and ignoring the variation of the main factor in the four-frequency moments near the origin $\left(\frac{1}{N_{i n t}}=0\right)$, then (8) is equivalent to (6), i.e. to the model of Jackson.

The details of the derivation are given in Appendix. It is noted that all the above equations derived are valid when the number of the independent samples $\mathrm{PRF}^{*} T_{\text {int }}$ caused by the radar pulse repetition frequency (PRF) is larger than the total number of the independent samples $N_{\text {tot }}$, i.e. $\mathrm{PRF}^{*} T_{\text {int }}>N_{\text {tot }}$.If we define an effective Doppler bandwidth $B_{d}=N_{\text {tot }} / T_{\text {int }}$, then the valid condition becomes $\mathrm{PRF}>B_{d}$. If $\mathrm{PRF}<B_{d}$, then $N_{\text {tot }}$ in (8a) should be modified to PRF* $T_{\text {int }}$.

\section{MODEL RESULTS FOR THE SPECKLE NOISE SPECTRUM}

In this section, we discuss results obtained from our model of speckle noise spectrum for different sea-state situations and for the case of an airborne spectrometer, with characteristics similar to those of the KuROS radar. This choice, without losing generality, enables us to compare the model results to observations (see section V). We extend the application of the model to other configuration in section VI.

The parameters in the following simulation are set as shown in Table I.

Table I RADAR PARAMETERS IN THE SIMULATIONS

\begin{tabular}{ll}
\hline Radar frequency & $13.5 \mathrm{GHz}$ \\
Central incidence & $13^{\circ}$ \\
$3 \mathrm{~dB}$ Beam width in azimuth, $\beta_{\phi}$ & $8.6^{\circ}$ \\
Radar range resolution & $1.5 \mathrm{~m}$ \\
Integration time & $33 \mathrm{~ms}$ \\
Platform velocity & $100 \mathrm{~m} / \mathrm{s}$ \\
Flight height & $2000 \mathrm{~m}$ \\
\hline
\end{tabular}

In section III.A, we first discuss the dependence of $P_{s p}(\mathrm{~K}, \Phi)$ with azimuth and the role of the different terms contributing to this dependence. In section III.B, we analyze the trend of the azimuthally-averaged speckle energy with wave number and more particularly the impact of sea-state conditions on this trend.

In the results presented below, $m_{t t}$ was calculated with (8i) and for various empirical ocean wave spectra $F(K, \Phi)$. For a pure wind wave situation, $F(K, \Phi)$ was chosen as the Elfouhaily spectrum - named EL [17] hereafter. A swell component was added in some simulations, with the swell spectrum expressed as a Gaussian function as in [18] 
Overall, three categories of cases of sea surface conditions were considered in our simulations. The parameters of the sea conditions for different categories are listed in Table II, including the wind speed $\mathrm{U}_{10}$, the wave inverse age $\Omega$, the peak wave length $\lambda_{p}$, the significant wave height Hs.

Table II

SEA SURFACE CONDITIONS USED FOR THE SIMULATION

\begin{tabular}{clll}
\hline & \multicolumn{1}{c}{$\begin{array}{c}\text { Case } 1 \\
\text { Pure wind wave }\end{array}$} & $\begin{array}{c}\text { Case } 2 \\
\text { Mixed sea }\end{array}$ & $\begin{array}{c}\text { Case } 3 \\
\text { Mixed sea }\end{array}$ \\
\hline Wind wave & $U_{10}=10 \mathrm{~m} / \mathrm{s}$, & $U_{10}=10 \mathrm{~m} / \mathrm{s}$, & $U_{10}=10 \mathrm{~m} / \mathrm{s}$, \\
component & $\Omega=0.84$ & $\Omega=0.84$ & $\Omega=0.84$ \\
& & $\mathrm{Hs}=2 \mathrm{~m}, \lambda_{p}=400$ & $\mathrm{Hs}=4 \mathrm{~m}, \lambda_{p}=200$ \\
Swell & & $\begin{array}{l}\text { m, propagating } \\
\text { along the wind } \\
\text { direction }\end{array}$ & $\begin{array}{l}\text { m, propagating } \\
\text { along the wind } \\
\text { direction }\end{array}$ \\
\hline
\end{tabular}

$\omega_{d}$ in (8i) is the wave scale limit which makes the quasispecular scattering approximation valid. We determined $\omega_{d}$ by the following method. At near-nadir incidence angles, the Physical Optics model, hereafter referred to as PO model, is considered accurate enough as long as polarization effects remain negligible, that is in the first 20 to $25^{\circ}$ incidence away from nadir [14], [19], [20]. Here PO is referred as the reference model. Firstly, we calculate the backscattering coefficients by PO and EL spectrum for the sea surface conditions. On the other hand, the backscattering coefficients by the approximation model-Quasi-specular scattering model [14] is expressed as:

$\sigma_{Q S}^{0}(\theta)=\frac{\left|R_{e}\right|^{2}}{m s s_{e}} \sec ^{4}(\theta) \exp \left(-\frac{\tan ^{2}(\theta)}{m s s_{e}}\right)$

$m s s_{e}=\int_{0}^{K_{d}} K^{2} F(K) d K$

Secondly, fitting (10) to $\sigma^{0}(\theta)$ values generated with the PO model in the incidence range of 0 to $18^{\circ}, R_{e}$ and $m s s_{e}$ can be inversed. Thirdly, we obtain $K_{d}$ according to (10) from the inversed $m s s_{e}$. Finally, $\omega_{d}$ is calculated by $\omega_{d}^{2}=K_{d} g$ for the case of deep water.

In order to estimate $N_{\text {int }}$, we calculate the modulation spectrum $P_{\text {mod }}(K, \Phi)$ due to the tilting waves as in [11]:

$P_{\text {mod }}(K, \Phi)=\frac{\sqrt{2 \pi}}{L_{\phi}}\left(\operatorname{ctg} \theta-\frac{\partial \ln \sigma^{0}}{\partial \theta}\right)^{2} K^{2} F(K, \Phi)$

where $\sigma^{0}$ is obtained by (9).

A. Variations with the look angle and impact of sea state conditions

From (8a) the energy of the speckle noise spectrum is determined by $N_{\text {tot }}(\Phi)$ for a given radar configuration. From (8b), $N_{\text {tot }}(\Phi)$ is a function of $N_{\text {surf }}, N_{\text {platf }}$, and $N_{\text {int }}$, where $N_{\text {surf }}$ is independent of the azimuth look direction, while $N_{\text {platf }}$, and $N_{\text {int }}$ change with it.

In this section we discuss the variations of $N_{\text {surf }}$ with the sea surface conditions, and those of $N_{\text {platf }}$ and $N_{\text {int }}$ with the azimuth look direction (as for an azimuthally scanning radar). For convenience, the relative look direction $\Phi$ is defined as $\Phi=\Phi_{1}-\Phi_{\text {platf }}$, where $\Phi_{1}$ is azimuth angle with respect to geographical North and the latter is the flight direction.

We first illustrate in Fig. 1 the variation of $N_{\text {surf }}$ with wind speed $U_{10}$ for three different values of the inverse wave age ( $\Omega=0.84,1$ and 2), corresponding to the fully developed, mature and young sea. As can be seen in Fig. $1, N_{\text {surf }}$ significantly increases with wind speed for all wave ages. For example, for fully-developed situations $N_{\text {surf }}$ increases from about 4 to about 13 over the wind speed range 6 to $18 \mathrm{~m} / \mathrm{s}$. As shows the trend with wind speed, the sensitivity of $N_{\text {surf }}$ to wind speed is much higher for fully-developed conditions than for young waves. From (8i), this is due to the variation of the velocity variance $m_{t t}$ with both wind and wave age.

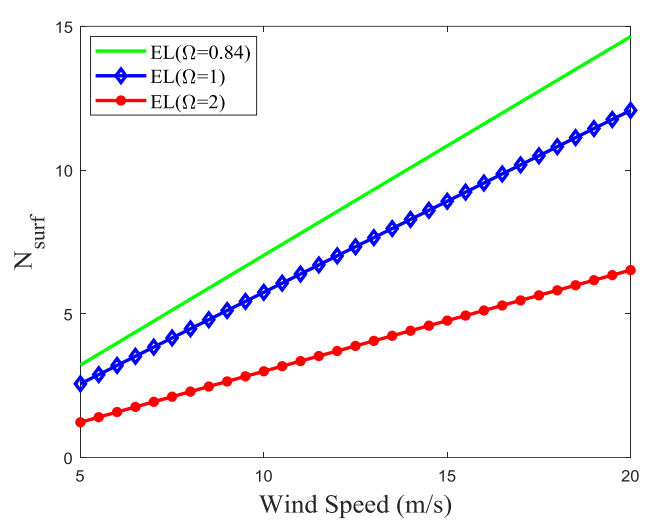

Fig. 1. $N_{\text {surf }}$ variation with wind speed under different inverse wave ages (green, blue, red, for respectively $0.84,1,2$ )

To illustrate the effect of swell on $N_{\text {surf }}$, Fig. 2 shows the variations of $N_{\text {surf }}$ with the significant height Hs of the swell component with the swell peak wavelength $\lambda_{p}=$ $150 \mathrm{~m}, 250 \mathrm{~m}, 350 \mathrm{~m}$. It shows that $N_{\text {surf }}$ increases with the swell Hs when keeping the wind wave component constant. When the swell Hs increases from $2 \mathrm{~m}$ to $7 \mathrm{~m}, N_{\text {surf }}$ is multiplied by $1.7,1.5,1.4$ for the swell peak wavelength $\lambda_{p}=$ $150 \mathrm{~m}, 250 \mathrm{~m}, 350 \mathrm{~m}$, respectively.

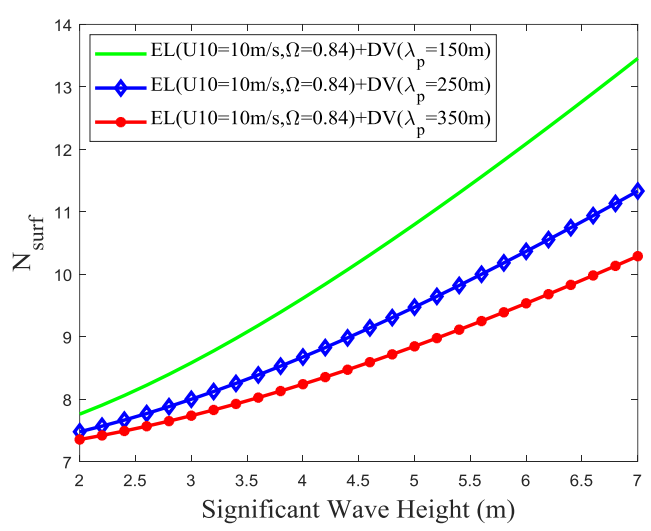

Fig. 2. $N_{\text {surf }}$ variation with wind speed for a mixed sea condition with fully developed wind waves and swell of different wavelengths (green, blue, red for 150,250 and $350 \mathrm{~m}$, respectively)

From Fig. 1 and 2, we draw a conclusion that $N_{\text {surf }}$ increases with wind speed and significant height Hs. The sensitivity with wind speed is much more important than with the significant height of a swell component. It is because that from (8e) $N_{\text {surf }}$ is determined by the sea surface condition through the velocity variance $m_{t t}$, which is dominated by the wind waves because of their higher surface velocities; the addition of a 'gentle' swell does not increase $m_{t t}\left(N_{\text {surf }}\right)$ significantly.

The parameter $N_{\text {int }}$ in (8f) is dependent on the sea conditions and on the radar azimuth looking angle with respect to the flight direction ( $8 \mathrm{~g}$ to $8 \mathrm{~h}$ ). Fig. 3 shows the variations of $N_{\text {int }}$ with the relative look direction $\Phi$, when the wind wave direction coincides with the flight direction, for a sea-state with a $10 \mathrm{~m} / \mathrm{s}$ fully developed wind wave situation (case 1) and for two cases of mixed sea with swell added to the wind sea (swell $\mathrm{Hs}=2 \mathrm{~m}$ and $4 \mathrm{~m}$ for cases 2 and 3 respectively). The swell direction was set aligned with the wind wave direction in cases 2 and 3 .

It shows that for each case, $N_{\text {int }}$ reaches maximum values in the cross-wave/cross-track direction, while it gets minimum values along the wave propagation direction which coincides in this case with the flight direction. This is because $N_{\text {int }}$ is proportional to $P_{\text {mod }}^{*} / m_{t t}$ according to (8f), where $P_{\text {mod }}^{*}$ is the sum of the modulation spectrum $P_{\text {mod }}$, which is related to the wave slope spectrum in (11) and a second term related to $m_{t t}$. 
This latter is much smaller than $P_{\text {mod }}$. Thus, $N_{\text {int }}$ is mainly dominated by the ratio $P_{\text {mod }} / m_{t t} . P_{\text {mod }}$ is maximum (minimum) along (cross) the wave propagation direction, thus $N_{\text {int }}$ is minimum (maximum) in this direction since $m_{t t}$ does not change with the azimuth anlge. Fig. 3 also shows that the sea-state condition impacts $N_{\text {int }}$ mainly in the along-wave direction with the smallest values observed for the highest significant wave height ( $N_{\text {int }}=40,30,10$ for the sea conditions of case 1 , case 2 and case 3 , respectively). In the cross-wave direction, the order of magnitude of $N_{\text {int }}$ is about 30 to 100 times higher than in the along wind direction.

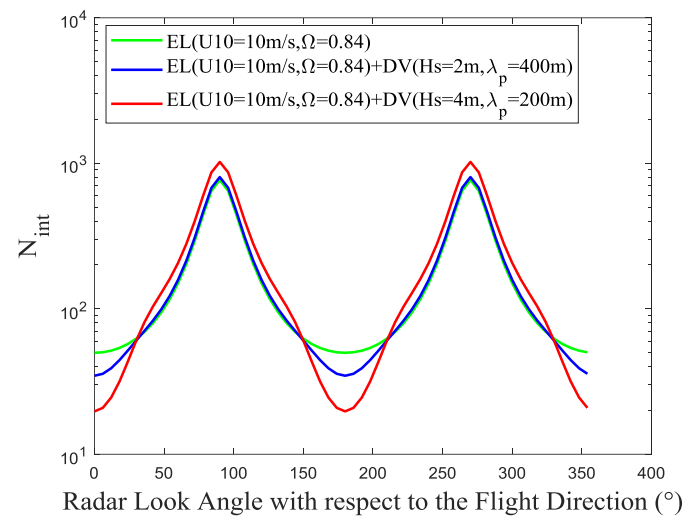

Fig. 3. $N_{\text {int }}$ as a function of the azimuth look direction (with respect to the flight direction) when the flight direction is aligned with wind-wave direction. The green, blue, and red curves are for sea surface conditions of cases 1 to 3 , respectively (see text)

From (8d) and (6) one notes that $N_{\text {platf }}$ is dependent only on the radar looking direction in azimuth with respect to the advection direction, whereas $N_{\text {tot }}$ varies with all parameters (flight direction, sea surface conditions and radar azimuth angle). Fig. 4 shows $N_{\text {platf }}$ and $N_{\text {tot }}$ variations with the relative azimuth looking direction for the pure wind wave case (case 1 , EL spectrum with $U_{10}=10 \mathrm{~m} / \mathrm{s}, \Omega=0.84$ ) when the flight track is perpendicular to the wind and wave directions. It shows that $N_{\text {platf }}$ is zero along the flight direction and reaches maximum values in the across flight direction. It is obvious from (6) since $N_{\text {platf }}$ is determined by $\sin \Phi$, where $\Phi$ is the azimuth angle relative to the radar flight direction. Fig. 4 also shows that along the flight direction (at $0^{\circ}$ and $180^{\circ}$ ), $N_{\text {tot }}$ is minimum, however, not equal to zero; it is because in this direction $N_{\text {tot }}$ is mainly determined by $N_{\text {surf }}$ along the flight direction (in this direction $N_{\text {platf }}$ is zero), and $N_{\text {int }}$ is big enough to avoid a null value of $N_{t o t}$. In contrast, in the across flight direction, just along the wind or wave direction, $N_{\text {int }}$ is minimum, and $N_{\text {tot }}$ is determined by both $N_{\text {platf }}$ and $N_{\text {int }}$.

Hence, in these conditions, the number of independent samples $N_{\text {tot }}$ is sufficient to avoid large value of the speckle noise spectrum when the antenna looks in the along-track direction.

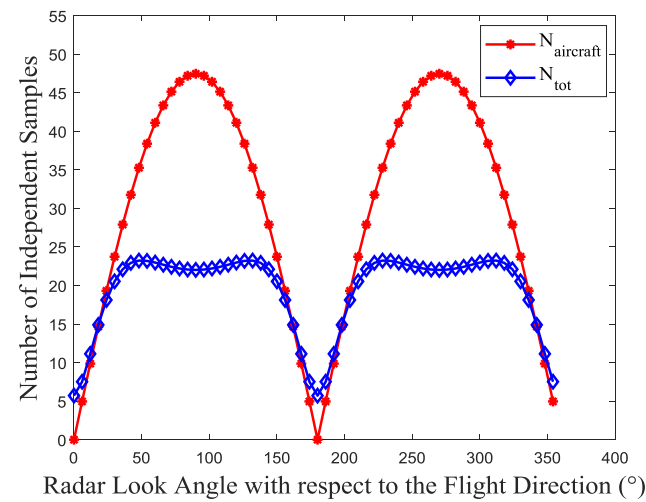

Fig. 4. Variation of $N_{\text {platf }}$ (in red) and $N_{\text {tot }}$ (in blue) with the azimuth look direction (with respect to the flight direction) when the flight direction is perpendicular to the wind-wave direction. The sea surface conditions are those of case 1 (see text).

Now we study the effect of the angle between the flight direction and the wind (or wave) direction on the number of the independent samples $N_{\text {tot }}(\Phi)$. Fig. 5 shows $N_{\text {tot }}(\Phi)$, for the same conditions as in Fig. 4 (fully developed wind waves), when the angle between the flight track and the wave direction is $0^{\circ}, 30^{\circ}, 60^{\circ}$ and $90^{\circ}$. It shows that $N_{\text {tot }}$ always reaches a maximum when the radar looks nearly perpendicularly to the flight direction, and is minimum when it looks along the flight direction. The minimum values of $N_{\text {tot }}$ is almost constant for different relative angles between the flight track and the wave direction. It is because along the flight direction, $N_{\text {tot }}$ is dominated by $N_{\text {surf }}$, which is independent of azimuth. In opposite, the maximum values of $N_{\text {tot }}$ decrease with relative angle between the flight track and the wave direction. For the developed wind wave with $U_{10}=10 \mathrm{~m} / \mathrm{s}$, the maximal $N_{\text {tot }}$ decreases from 44 to 22 when this angle changes from $0^{\circ}$ to $90^{\circ}$. When the wave direction coincides with the flight direction, both $N_{\text {mov }}$ and $N_{\text {int }}$ reach their maximum values in the same direction. When the relative angle between the flight track and the wave direction increases, the angle between the direction where $N_{\text {mov }}$ is maximum and $N_{\text {int }}$ is maximum also increases, which results in a lower value of the maximum value of $N_{\text {tot }}$ and a more stable value of $N_{\text {tot }}$ with look angle outside a sector of $\pm 30^{\circ}$ along-track.

In summary, the behavior of the total number of independent samples, $N_{\text {tot }}$, has a multi-harmonic shape with minimum of $N_{\text {tot }}$ always in the along-track direction and a minimum value which depends on sea-state. The azimuth position of the maximum of $N_{\text {tot }}$ varies with angle between the flight direction and wave propagation direction, and its level is also dependent of both sea-state and relative direction between waves and flight.

In order to study the role of $N_{\text {int }}$ in this general behavior of $N_{\text {tot }}$, we define the ratio $R_{\text {int }}$ of the contribution of $N_{\text {int }}$ to the total speckle noise spectrum $P_{s p}$ :

$R_{\text {int }}=\frac{\frac{1}{N_{\text {int }}}}{\frac{1}{N_{\text {int }}}+\frac{1}{N_{\text {platf }}}}=\frac{N_{\text {platf }}}{N_{\text {int }}+N_{\text {platf }}}$

This ratio indicates the impact of non-neglecting the variation of the main factor in the four-frequency moment near the origin in the development of speckle noise spectrum while considering a moving surface.

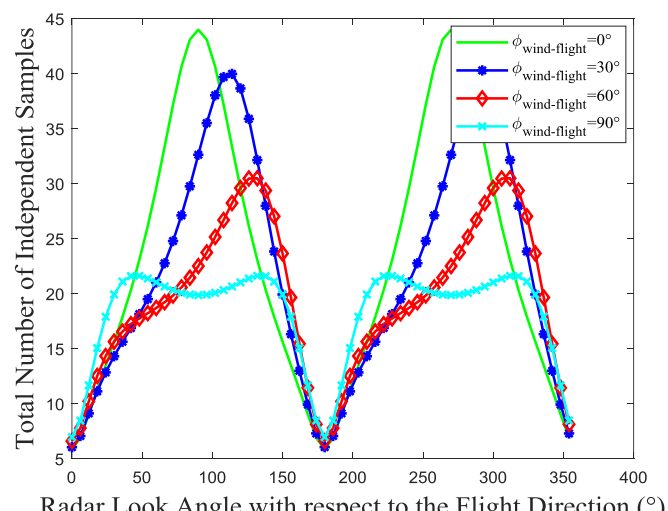

Fig. 5. $N_{\text {tot }}$ as a function of the azimuth look direction (with respect to the flight direction) for different flight angles with respect to the wave direction: green, blue, red, yellow for $0^{\circ}, 30^{\circ}, 60^{\circ}, 90^{\circ}$, respectively. The sea surface conditions are those of case 1 (fully developed wind waves). 


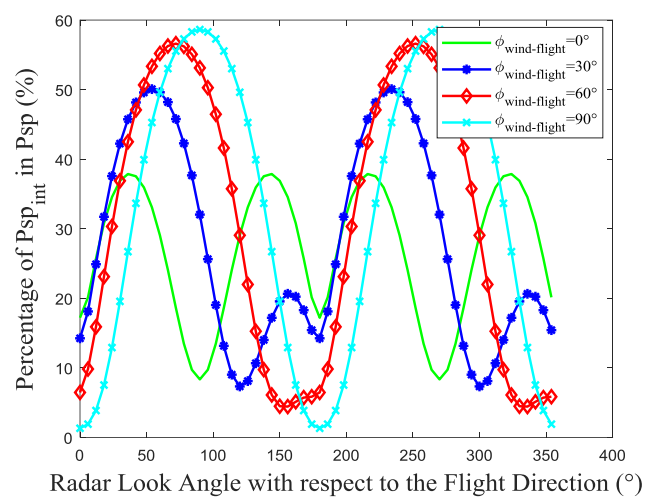

Fig. 6. $R_{\text {int }}$ as a function of the relative look direction with the color code and sea surface conditions similar to Fig. 5.

For the same sea conditions as in Fig. 5, Fig. 6 shows $R_{\text {int }}$ as a function of the radar azimuth look angle (with respect to the flight direction) for different wave propagation directions. It shows that $R_{\text {int }}$ ranges between almost $0 \%$ and $60 \%$ depending on the azimuthal look angle. It is minimum in the cross-wave direction for any flight directions. It is because from (8f) and (8h), when observing in the direction perpendicular to the wave propagation $\mathrm{P}_{\text {mod }}$ approaches zero, which leads to large $N_{\text {int }}$ values. Fig. 6 shows that the maximum values of $R_{\text {int }}$ change from $38 \%$ to $58 \%$ when the flight direction goes from $0^{\circ}$ to $90^{\circ}$ with respect to the wave propagation direction. When this angle is $90^{\circ}$ (cyan curve in Fig. 6), $\mathrm{R}_{\text {int }}$ is maximum in the along wave propagation direction. It is because, for an angle of $90^{\circ}$ between the flight direction and the waves, $P_{\text {mod }}$ (or $\left.\left(N_{\text {int }}\right)^{-1}\right)$ approaches to its maximum, whilst $\left(N_{\text {platf }}\right)^{-1}$ is minimum. For angles different from $90^{\circ}$, there is no direction where $\left(N_{\text {int }}\right)^{-1}$ and $\left(N_{\text {platf }}\right)^{-1}$ are simultaneously maximum and minimum, respectively, so that $R_{\text {int }}$ peak values cannot get the same maximum as for the case of angle of $90^{\circ}$. When changing from $90^{\circ}$ to $0^{\circ}$ the locations of the maximum of the curves are shifted to the cross-wave direction. For flight directions of 0 and $30^{\circ}$ with respect to the wave direction, the variation with the direction is more complex with secondary maxima appearing. A maximum value of almost $60 \% R_{\text {int }}$ as found in these fully developed conditions indicate that the term $N_{\text {int }}$ as taken into account in our model is not negligible for certain conditions of observations.

\section{B. Omni-directional speckle noise spectrum}

In this section, we analyze the omni-directional spectrum of speckle noise as a function of the surface wavenumber $K$, and its dependence with wind speed, sea-state and radar configurations.

The omni-directional speckle noise spectrum is defined as: $P_{s p}(K)=\int_{0}^{2 \pi} P_{s p}(K, \Phi) d \Phi$

Besides the density of the speckle noise spectrum, we also focus on a Signal-To-Noise ratio (SNR), which is defined as the ratio of the signal spectrum $P_{1}(K, \Phi)$ in (A17) and the noise spectrum $P_{s p}(K, \Phi)$ in $(8 \mathrm{a})$,

$\operatorname{SNR}(K, \Phi)=2 \pi K_{p} N_{\text {tot }}(\Phi) \operatorname{tri}\left(\frac{K}{2 \pi K_{p}}\right) P_{\text {mod }}(K, \Phi)$

Accordingly, an azimuthally-integrated SNR value is defined as:

$\operatorname{SNR}(K)=\frac{1}{2 \pi} \int_{0}^{2 \pi} \operatorname{SNR}(K, \Phi) d \Phi$

Firstly, we study the effect of wind speed on $P_{s p}(K)$, based on our model with the EL wave spectrum used as input with a prescribed wind speed $U_{10}$ and wave age $\Omega$.

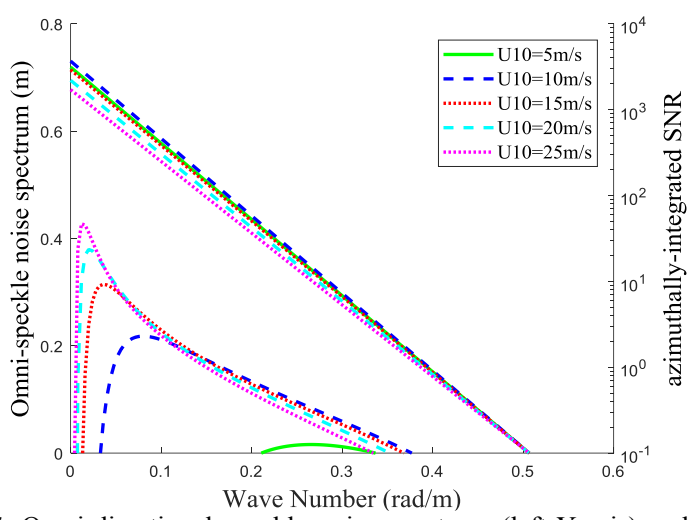

Fig. 7. Omni-directional speckle noise spectrum (left Y-axis) and SNR (right Y-axis) as a function of the wavenumber $K$ for different wind speeds $U_{10}=5,10,18 \mathrm{~m} / \mathrm{s}$ and for fully developed wind waves

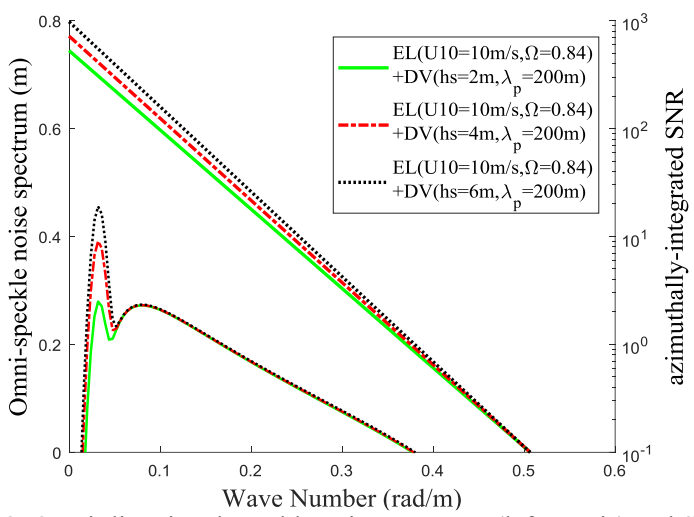

Fig. 8. Omni-directional speckle noise spectrum (left Y-axis) and SNR (right Y-axis) as a function of the wavenumber $K$ for the mixed sea, with the different Hs for the swell component

Fig. 7 shows $P_{s p}(K)$ (left Y-axis) and SNR $(K)$ (right Y-axis) for different wind speed values (taken at the height of $10 \mathrm{~m}$ ), namely every $5 \mathrm{~m} / \mathrm{s}$ from 5 to $25 \mathrm{~m} / \mathrm{s}$ and with an inverse wave age of 0.84 (fully-developed waves). It shows that the energy of the speckle spectrum is not very sensitive to the wind speed. The relationship between the speckle energy and the wind speed is not strictly monotonous: the energy for $5 \mathrm{~m} / \mathrm{s}$ is a little lower than that for $10 \mathrm{~m} / \mathrm{s}$, but is a little larger than that for $15 \mathrm{~m} / \mathrm{s}$. As the wind speed continues to increase from 15 to 25 $\mathrm{m} / \mathrm{s}$, both the value at the origin $(K=0)$ and the slope of the trend with $K$ decreases. The change of $P_{s p}(K)$ with wind speed is due to the azimuthally averaged $N_{\text {tot }}$ factor, which is dependent of wind speed through the terms in $N_{\text {surf }}$ and $N_{\text {int }}$. As described in section III.A, $N_{\text {surf }}$ is determined by the velocity variance of the sea surface $m_{t t}$, while $N_{\text {int }}$ is dominated by the ratio of the modulation spectrum $P_{\text {mod }}$ in (11) and $m_{t t}$. With wind speed increasing, on one hand $m_{t t}$ and $N_{\text {surf }}$ increase, but on the other hand $N_{\text {int }}$ decreases because $P_{\text {mod }}$ increases more than $m_{t t}$ does. The combination of these effects (enlarging $N_{\text {surf }}$ but reducing $N_{\text {int }}$ ) lead to a not monotonous dependence of speckle energy with wind speed. Fig. 7 also shows that SNR increases greatly with the wind speed, the SNR maximum appear at the peak wave number.

We also studied the effect of the developing stage of the wind waves. Our results indicate (not shown here) that $P_{s p}(K)$ decreases slightly with the inverse wave age for the same wind.

The impact of an additional swell is illustrated in Fig. 8, which shows $P_{s p}(K)$ for a mixed sea, with the wind wave component for $U_{10}=10 \mathrm{~m} / \mathrm{s}$, inverse wave age $\Omega=0.84$ and a swell component with a peak wavelength $\lambda_{p}=200 \mathrm{~m}$ and a significant height $H s=2 \mathrm{~m}, 4 \mathrm{~m}$, and $6 \mathrm{~m}$. It shows that the energy of the noise spectrum is not very sensitive to the swell 
wave height, when all other parameters are kept constant, $P_{s p}(K)$ increases slowly but monotonously when Hs of the swell component increases from $2 \mathrm{~m}$ to $6 \mathrm{~m}$. It is because, as described in section III.A, $m_{t t}\left(N_{\text {surf }}\right)$ is dominated by the wind waves because of their higher frequencies, while the addition of the swell does not increase $m_{t t}\left(N_{\text {surf }}\right)$ significantly. On the other hand, from (8f), since $m_{t t}$ does not change a lot $N_{\text {int }}$ is dominated by the modulation spectrum $P_{\text {mod }}$, which is sensitive to the long waves. Thus, the addition of swell increases $P_{\text {mod }}$, and accordingly decreases $N_{\text {int }}$. The combination of the two effects (enlarging $N_{\text {surf }}$ a little but reducing $N_{\text {int }}$ ), leads to a monotonous increase of the energy of the noise spectrum with Hs of the swell.

We also studied the effect of different peak wavelengths of the swell component. Our results indicate (not shown here) that $P_{s p}(K)$ increases very slightly with the swell $\lambda_{p}$ when the other parameters are kept constant. Thus, for swell the speckle is almost not affected by its dominant wavelength (or period).

In summary, for the radar configuration chosen here the azimuthally-integrated value of the speckle noise spectrum is not very sensitive to the sea surface conditions because the effects of the sea conditions on the density of speckle noise spectrum by $N_{\text {surf }}$ and $N_{\text {int }}$ are opposite and comparable.

We will see in section VI, that the conclusion may change when considering other radar frequencies, central incidences, $3 \mathrm{~dB}$ beam width in azimuth, platform speed and platform height.

\section{EXPERIMENTAL ESTIMATE OF THE SPECKLE NOISE SPECTRUM}

Two empirical methods have been proposed and implemented in the past for estimating the speckle noise spectrum from the backscattered intensity observations themselves: the "post-integration" method [3] and the crossspectrum method first applied on SAR data [2] and then adapted to the real-aperture scanning configuration [10]. The two methods require that the radar samples correspond to overlapping sea surface areas for adjacent integration times. With the observation configuration of the airborne KuROS radar [10] both methods, either based on a cross-spectrum analysis or on different post-integrated signal are possible. Here, we choose the method based on different post-integrated signals because it optimizes the overlapping areas of the different signal samples used to estimate the speckle noise spectrum.

We recall first that KuROS is a $\mathrm{Ku}$-band $(\mathrm{f}=13.5 \mathrm{GHz})$ radar, with a near-nadir pointing rotating antenna, which provides the backscattering coefficients in the incidence range from about $5^{\circ}$ to $18^{\circ}$ and the azimuth range of 0 to $360^{\circ}$. The data sets analyzed here come from KuROS flights carried out in the northwestern part of the Mediterranean Sea as part of the Hymex experiment [10], [12].

A speckle spectrum estimation $P_{s p}(K, \Phi)$ can be derived from the measured radar cross-section $\sigma^{0}$ fluctuation spectra. Let $\delta \sigma^{0}(\mathrm{x}, \Phi)$ be the fluctuation function of the measured $\sigma^{0}$ along the horizontal axis $\mathrm{x}$ (taken as aligned with the incidence plane) in the azimuth direction, and $P_{\sigma^{0}}(K, \Phi)$ its spectral density as a function of the wave number modulus in the azimuth direction. Then, as shown in (A39) $P_{\sigma^{0}}(K, \Phi)$ is given by:

$P_{\sigma^{0}}(K, \Phi) \cong P_{I R}(K, \Phi) P_{\text {mod }}(K, \Phi)+P_{s p}(K, \Phi)$

In (16), $P_{\sigma^{0}}(K, \Phi)$ is obtained in each direction $\Phi$ from the radar data, as the power spectrum of the relative fluctuations along the $\mathrm{x}$ axis (look direction) of $\sigma^{0}$. In order to obtain the speckle noise spectrum $P_{s p}(K, \Phi)$, from this expression, we used the post-integration method proposed in [3]. This method makes use of $\sigma^{0}$ values estimated - for the same raw data- over two different integration durations ( $T_{\text {int }}$ and $N \cdot T_{\text {int }}$ ) and assumes that the speckle reduction between the two cases ( $T_{\text {int }}$ and $N \cdot T_{\text {int }}$ ) is of a factor $N$. Combining the spectrum of signal fluctuation calculated over the period of $N \cdot T_{\text {int }},\left(P_{\sigma^{0}}\right)_{N T \text { Tint }}$, and the one resulting from the average of $N$ spectra each calculated over the period of $T_{\text {int }},\left\langle\left(P_{\sigma^{0}}\right)_{\text {Tint }}\right\rangle_{N}$, and assuming that the wave contribution $P_{\text {mod }}$ is identical in the two cases, $P_{s p}(K, \Phi)$ can be obtained:

$P_{s p}(K, \Phi)=\frac{N}{N-1}\left(\left\langle\left(P_{\sigma^{0}}(K, \Phi)\right)_{\text {Tint }}\right\rangle_{N}-\left(P_{\sigma^{0}}(K, \Phi)\right)_{N T \text { int }}\right)$

Here for KuROS, we used $T_{\text {int }}=33 \mathrm{~ms}$ and $N=3$.

We recall here that KuROS range resolution is $1.5 \mathrm{~m}$, leading to a horizontal resolution at the central incidence $\left(13.5^{\circ}\right)$ of $\approx$ $6.5 \mathrm{~m}$. In the analysis presented here below, the spectral analysis is carried out by considering the horizontal footprint covered by the $8^{\circ}$ to $18^{\circ}$ incidence range, i.e., at $\pm 5^{\circ}$ from the central incidence angle $13^{\circ}$. This corresponds to a footprint of $475 \mathrm{~m}$ or $710 \mathrm{~m}$ for the standard flight levels of $2000 \mathrm{~m}$ and $3000 \mathrm{~m}$, respectively.

The energy density spectra are binned in 64 wavenumbers and 60 azimuth directions. By fitting each azimuthal estimate of $P_{s p \_m e a s}(K, \Phi)$ to the functional shape $(8 \mathrm{a})$, we obtain an empirical estimate of the total number of independent samples $N_{\text {tot_meas }}(\Phi)$ and of $K_{p}(\Phi)$. By averaging $K_{p}(\Phi)$ over the azimuth angles $\left(0\right.$ to $\left.360^{\circ}\right)$, we obtain a mean $K_{p}$, and hence an estimate of the horizontal effective resolution $\delta x=\left(K_{p}\right)^{-1}$.

The results on $N_{\text {tot_meas }}(\Phi)$ and their omni-directional counterpart (estimated from (13)) are shown in Fig. 10 and 9, respectively for 9 different situations. They are discussed together with the model results in the next section.

\section{VALIDATION OF THE MODEL OF SPECKLE NOISE SPECTRUM}

In this section, we use the estimates of speckle noise spectrum from KuROS to validate the model presented in section III. In total, there are $12 \mathrm{KuROS}$ flights with coincident wind and wave measurements from the 'Lion' buoy (see [10]). From this data set, we have rejected in our analysis 3 cases (flights 9, 16 and 22) because it turned out that for these flights $\sigma^{0}$ values are more than $5 \mathrm{~dB}$ below normal, which leads in an abnormal speckle noise spectrum. For flight 4 and 11, the plane flew twice over the buoy with different flight directions. Table III lists for the 11 cases analyzed hereafter, the wind and wave parameters measured from the 'Lion' Buoy, namely the wind speed at $10 \mathrm{~m}$ height $U_{10}$, wind direction $\varphi_{\text {wind }}$, the significant height Hs, the peak frequency $f_{p}$, wave direction $\varphi_{\text {wave }}$. In the same table, the inverse wave age $\Omega$ is listed to indicate the developing stage of the wind waves. Here $\Omega$ is calculated as $\Omega=U_{10} \sqrt{k_{p} / g}$, where $k_{p}$ is the peak wavenumber is related to $f_{p}$ by the dispersion relationship in deep water $k_{p} g=\left(2 \pi f_{p}\right)^{2}$.

The omni-directional speckle noise spectrum $P_{s p \_m o d}(K)$ from the model was estimated using (8a) to (8i) with $K_{p}$ estimated from the empirical $\delta x$ value; the wind and wave parameters shown in Table I were used to estimate the velocity variance $m_{t t}$ which affects the $N_{\text {int }}$ and $N_{\text {surf }}$ terms according to $(8 \mathrm{e})$ and $(8 \mathrm{f})$. For that, a wind wave spectrum following the Elfouhaily's expression was calculated for the observed wind speed and wave age at the buoy location. The modulation spectrum $P_{\text {mod }}$ which affects the $N_{\text {int }}$ term (8f) was taken as provided by the Kuros data processing. $k_{d}$ in (8i) was determined following the method described in section III. 
We also calculated $P_{s p_{-} J a c}^{\prime}(K)$ and $N_{J a c}^{\prime}(\Phi)$ by using (6) which come from Jackson's model [11], and using the same input parameters $K_{p}$ and $P_{\text {mod }}$ described just above. $\mu$ was estimated by using (7).

Finally we compared $P_{s p}(K)$ and $N_{\text {tot }}(\Phi)$ predictions from our model, $P_{s p_{-} J a c}^{\prime}(K)$ and $N_{J a c}^{\prime}(\Phi)$ predictions from Jackson 's model in [11], and $P_{\text {sp_meas }}(K)$ and $N_{\text {tot_meas }}(\Phi)$ estimated from the KuROS data. In order to estimate the error between the measurements and model values, an Average Relative Error is defined as:

$\mathrm{ARE}=\frac{1}{N} \sum_{i=1}^{N}\left|\frac{V_{i \_ \text {meas }}-V_{i \_ \text {modl }}}{V_{i_{\_} \text {modl }}}\right|$

Where $V_{i_{\text {meas }}}$ are the measurements of $P_{s p}(K)\left(\right.$ or $\left.N_{\text {tot }}(\Phi)\right)$ at
$K_{i}\left(\right.$ or $\left.\Phi_{i}\right), K_{i}=i \Delta K, \Phi_{i}=i \Delta \Phi, \Delta K=\frac{1}{64} K_{\max }, K_{\max }=\frac{\pi}{\delta x}$, $\delta x \approx 6.5 \mathrm{~m}, \Delta \Phi=6^{\circ}, V_{i_{\text {modl }}}$ are the calculation results from the model presented in section II. Considering the theoretical horizontal resolution $\delta x$ is about $6.5 \mathrm{~m}, \mathrm{KuROS}$ can only detect the waves with the wavelength larger than at least 2 times the horizontal resolution. Thus, the detectable maximum $K_{\text {detc } \_ \text {max }} \approx 0.24$. On the other hand, with the limit of $K L_{y} \gg$ $1[1], L_{y}=\frac{L_{y}^{*}}{2 \sqrt{2 \ln 2}}, L_{y}^{*}=2 * H * \tan \left(\frac{\beta_{\phi}}{2}\right) * \cos ^{-1} \theta$, where $H$ is the flight height, $\beta_{\phi}=8.6^{\circ}$, the minimal detectable $K_{\text {detc_min }}$ is set as $0.038 \mathrm{rad} / \mathrm{m}$. Therefore, $K_{i}$ is between $K_{\text {detc_min }}$ and $K_{\text {detc_max }}$.

Table. III SEA CONDITIONS FOR DIFFERENT FLIGHTS FROM BUOY "LION"

\begin{tabular}{ccccccccccc}
\hline Flight No. & $\begin{array}{c}\mathrm{U} 10 \\
(\mathrm{~m} / \mathrm{s})\end{array}$ & $\begin{array}{c}\varphi_{\text {wind }}\left({ }^{\circ} /\right. \\
\mathrm{N})\end{array}$ & $\begin{array}{c}\mathrm{Hs} \\
(\mathrm{m})\end{array}$ & $\begin{array}{c}f_{p} \\
(\mathrm{~Hz})\end{array}$ & $\begin{array}{c}\varphi_{\text {wave }} \\
(\% / \mathrm{N})\end{array}$ & $\Omega$ & $\begin{array}{c}\varphi_{\text {flight }} \\
(\% / \mathrm{N})\end{array}$ & $\begin{array}{c}\left|\varphi_{\text {wave }}-\varphi_{\text {flight }}\right| \\
\left({ }^{\circ}\right)\end{array}$ & $\begin{array}{c}\text { Flight } \\
\text { speed }(\mathrm{m} / \mathrm{s})\end{array}$ & $\begin{array}{c}\text { Flight height } \\
(\mathrm{m})\end{array}$ \\
\hline 4- leg 1 & 13.65 & 285 & 2.8 & 0.135 & 315 & 1.182 & 48 & 87 & 99.2 & 2072.7 \\
4- leg 2 & & & & & 312 & & 142 & 10 & 114 & 2072.9 \\
11- leg 1 & 12.85 & 325 & 3.1 & 0.135 & 306 & 1.112 & 45 & 81 & 100.9 & 2195.8 \\
11- leg 2 & & & & & 318 & & 143 & 5 & 115 & 2199.1 \\
13 & 17.5 & 320 & 5.4 & 0.100 & 306 & 1.118 & 185 & 59 & 102.8 & 2916.2 \\
14 & 15.15 & 70 & 4.75 & 0.111 & 72 & 1.078 & 106 & 34 & 92.1 & 2135.9 \\
15 & 11.55 & 350 & 2.65 & 0.117 & 6 & 0.868 & 266 & 80 & 112.6 & 3141.9 \\
17 & 14.15 & 100 & 4.7 & 0.111 & 72 & 1.010 & 166 & 86 & 85.6 & 2130.3 \\
18 & 9.8 & 120 & 5.2 & 0.088 & 60 & 0.89 & 145 & 85 & 92.6 & 2031.7 \\
20 & 17.2 & 140 & 3.65 & 0.117 & 336 & 1.29 & 188 & 32 & 110.5 & 2922.1 \\
21 & 19.5 & 300 & 6.05 & 0.100 & 285 & 1.245 & 269 & 16 & 87.0 & 2034.2 \\
\hline
\end{tabular}
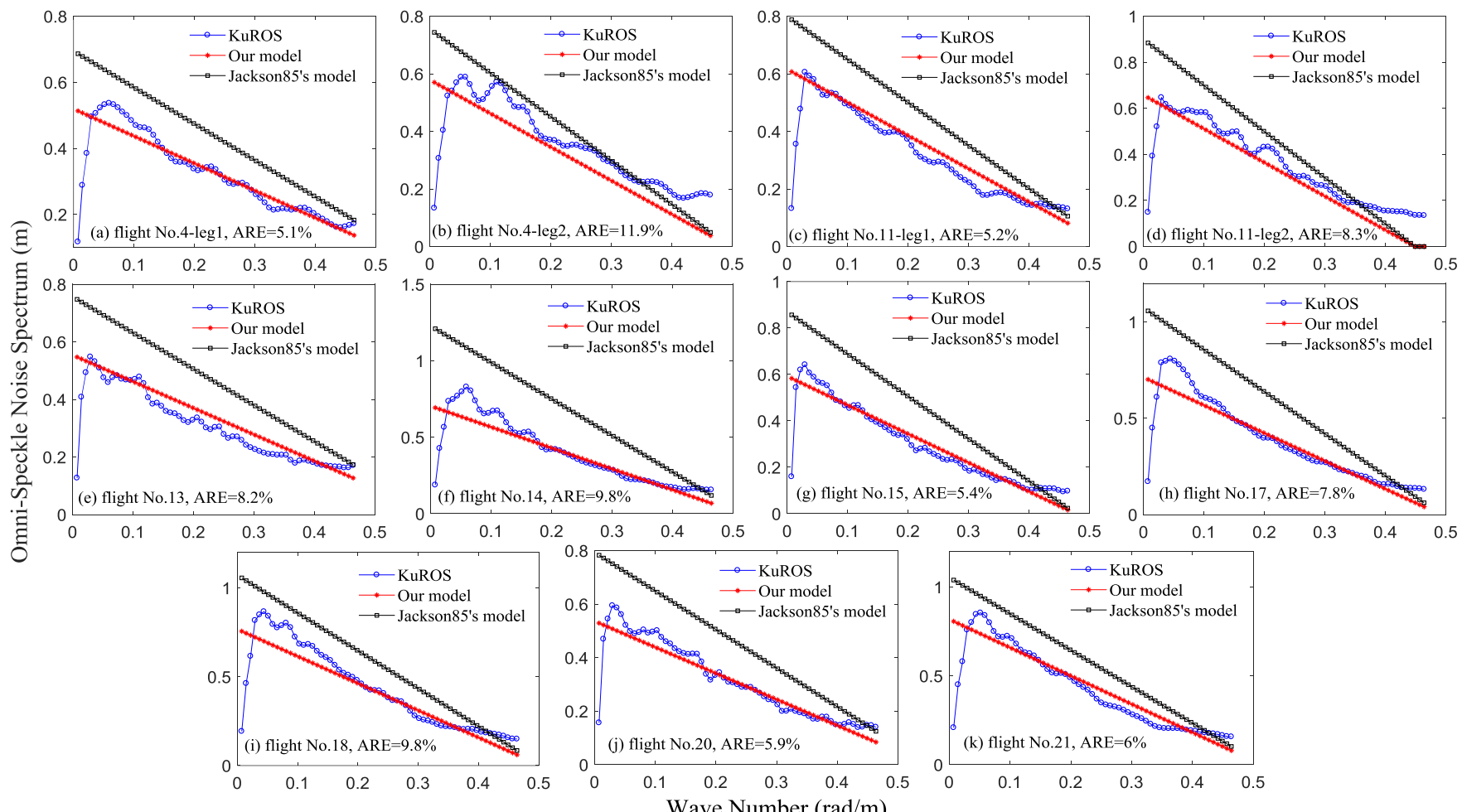

Fig. 9. Omni-directional speckle noise spectrum as a function of wavenumber for the cases listed in Table III: panels (a, b, c, d, e, f, g, h, i, j, k) refer respectively to flights 4-leg1, 4-leg2, 11- leg 1, 11-leg 2, 13, 14, 15, 17, 18, 20, 21. The blue, red and black curves are for respectively, the empirical estimates from KuROS data, from our model, and from Jackson's model

Fig. 9 (a) shows the omni-directional speckle noise spectra for the flight 4-leg 1, when the flight direction was nearly perpendicular to the wave direction. It shows that our model (red line) agrees well with the empirical estimates of the omnidirectional speckle noise spectrum (blue line), with the error ARE below 5\%, while Jackson's model (black line) overestimates the omni-directional speckle noise spectrum at all $K$ and overestimates the decreasing trend with $K$. Fig. 10 (a) shows for the same cases, $N_{\text {tot }}(\Phi)$ as a function of the observation azimuth angle with respect to the flight direction. It shows that $N_{\text {tot }}(\Phi)$ from our model, and from Jackson's model, both show the minimum values at about the flight direction, in agreement with the empirical estimates. This was expected because at the flight direction, the value of $N_{\text {platf }}$ approaches 1 . In this along-track direction, $N_{\text {tot }}$ from our model is in better agreement with the empirical estimates than the result from Jackson's model, because it is determined by $N_{\text {surf }}$ proposed in our model. In contrast, for Jackson's model 
the time-varying properties of the sea surface are ignored which induces an underestimate of $N_{t o t}$ in the along-track direction. It proves that the $N_{\text {surf }}$ term as added in our model is necessary to guarantee consistency, especially for look angles aligned with the flight direction. In a wide sector around the acrossflight direction, $N_{\text {tot }}$ from the Jackson's model is overestimated with respect to the empirical estimation. This is due to the omitted contribution of $N_{\text {int }}$ in the Jackson's model which comes from neglecting the variation of the main factor in the four-frequency moment near the origin. It proves that the term $\left(N_{\text {int }}\right)^{-1}$ added in our model is also necessary to guarantee consistency with observations. In contrast Fig. 10 (a) shows that $N_{\text {tot }}(\Phi)$ calculated with our model better agrees with the empirically estimated $N_{\text {tot }}(\Phi)$ over all azimuth angles. The small remaining differences between the measured $N_{\text {tot }}$ and that calculated with our model may come from the statistical fluctuations of the measured $N_{\text {tot }}$ (here we estimated $N_{t o t}$ as averaged values over $6^{\circ}$ bins in azimuth) or small remaining uncertainties on the geometry (incidence angle, azimuth angle).

The other panels in fig. 9 and fig. 10 show the omnidirectional speckle noise spectrum and $N_{\text {tot }}$ for flights 11,13 , $14,15,17,18,20,21$ respectively, from which the same conclusions as those discussed in details for flight 4 can be drawn. The errors between the measured omni-directional speckle noise spectrum and that estimated by our model are all below $10 \%$. The minimum values of $N_{\text {tot }}$ predicted by our model is of about 8 to 10 (Fig. 10) and agree well with the experimental values. The maximum values of $N_{\text {tot }}$ are within range 25 to 50 , depending of the flight conditions.

It is interesting to note the different shapes of the $N_{t o t}$ curves for different flight directions with respect to the wave direction. When the flight direction is aligned with the wave propagation direction (Fig. 10(b, d, k)), both the KuROS estimates and our model indicate a well-marked peak in the cross-track directions. This is very similar to the shape of the green curve in Fig. 5, namely the $N_{\text {tot }}$ curve simulated with our model with the flight-wave angle set as zero. In these conditions, azimuth variations from Jackson's model remains close to the observations and to our model.

On the other hand, for the files when the flight direction is nearly perpendicular to the wave direction (Fig. 10(a, c, g,h, i)), instead of a well-shaped peak, a large plateau or a multi-peak signature is observed in the $N_{\text {tot }}$ curves around the cross-wave direction. This is very similar to the shapes of the blue curve in Fig. 5, namely the $N_{\text {tot }}$ curve simulated with our model with the flight-wave angle set as $90^{\circ}$. In these cases, the discrepancies with respect to Jackson's model are the largest.

Overall, the comparisons with the empirical estimates of the density spectrum show that our model, in opposite to Jackson's model is able to reproduce the main trend of the speckle density spectrum variations with azimuth and with wave number in a large variety of conditions. This validates our model and indicates that it is essential to consider a moving surface in the speckle model and to avoid over simplification in the expression of the scattering matrix moments.
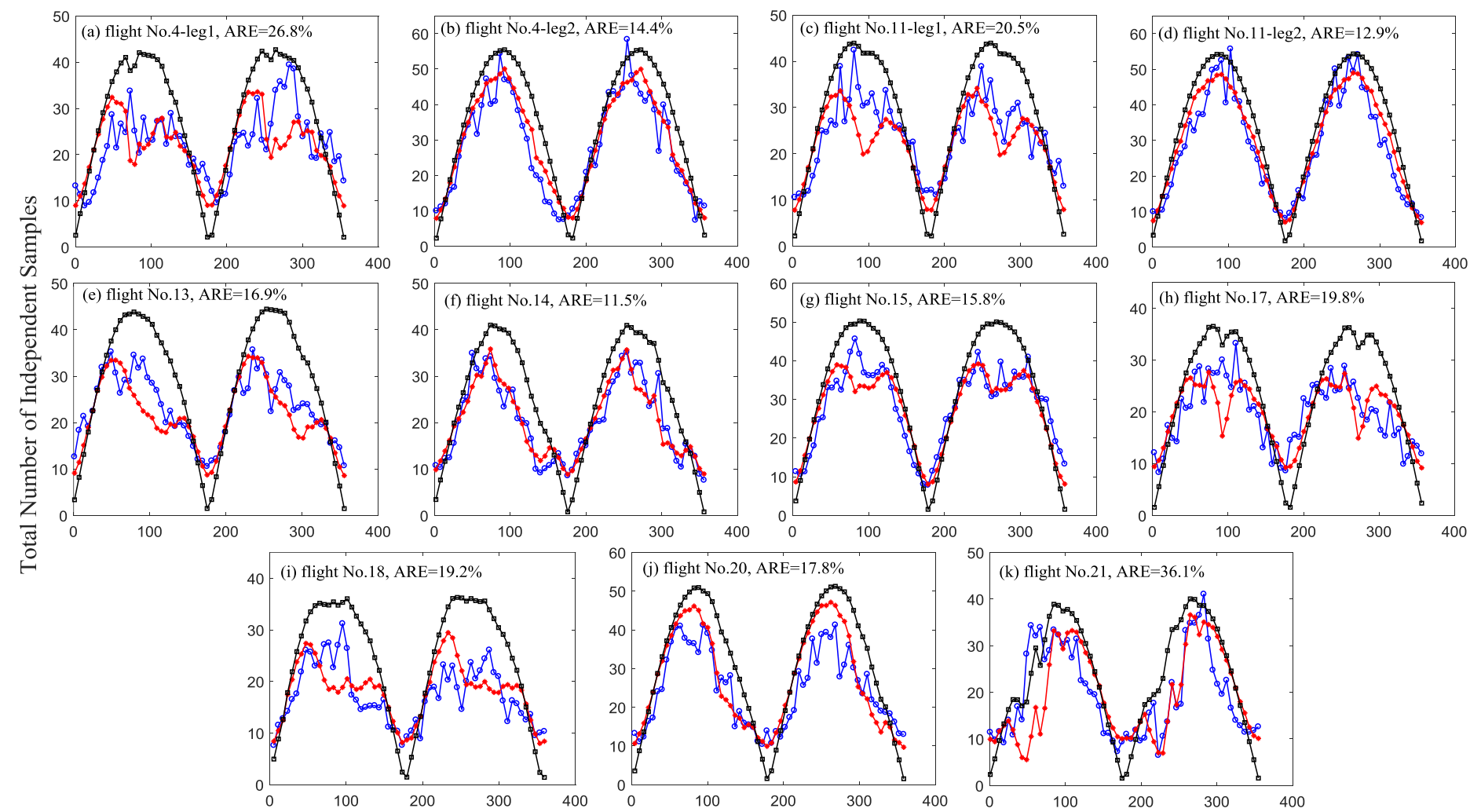

Radar Look Angle with respect to the Flight Direction $\left({ }^{\circ}\right)$

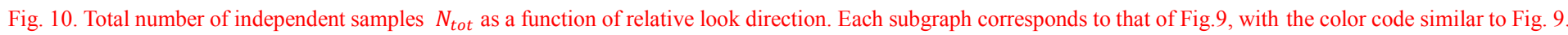

\section{INFLUENCE OF RADAR CONFIGURATION ON THE SPECKLE SPECTRUM}

Now that the model of speckle is validated under its limit of application (near-nadir incidence) we propose to extend its use to other configurations of observations while staying in the near-nadir configuration. In this section, we study the impact of the radar configurations on omni-directional speckle noise spectrum.

Firstly, we examine the impact of the platform advection speed. Then we analyze the impact of the footprint dimension in azimuth (either by changing the beam aperture or the flight height). Finally, we discuss the influence of radar frequency and of incidence angle.

When we increase the platform speed, the azimuthallyaveraged $N_{\text {mov }}$ term increases, which leads to the larger 
contribution of $N_{\text {int }}$ and smaller contribution of $N_{\text {surf }}$. Fig. 11 show the omni-directional speckle noise spectrum when the flight speed is $300 \mathrm{~m} / \mathrm{s}$ and other parameters are the same as those in Fig. 7 and Fig. 8, respectively.

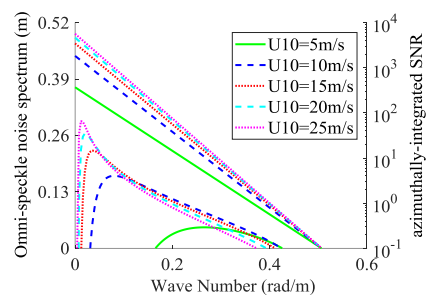

(a)

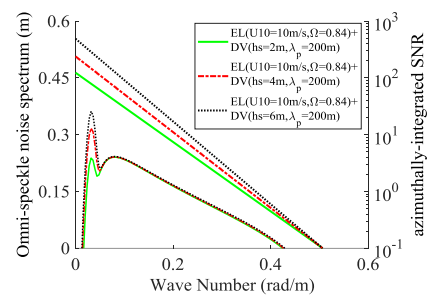

(b)
Fig. 11. Omni-directional speckle noise spectrum (left Y-axis) and SNR (right Y-axis) as a function of the wavenumber $\mathrm{K}$ for the flight speed of 300 $\mathrm{m} / \mathrm{s}$, the sea surface conditions are the same as those of Fig. 7 and Fig. 8 for (a) and (b), respectively.

From Fig. 11, it is observed that in this configuration, the density of omni-directional speckle noise increases monotonously and significantly with wind speed or Hs of swell. It is because in such cases, $N_{\text {int }}$ dominates the density of the spectrum. The increase of the wind speed and Hs lead to a larger increase of $P_{\text {mod }}$ than of $m_{t t}$, and a decrease of $N_{\text {int }}$, which contribute to the increase of the density of the spectrum. Compared with Fig. 7 and Fig. 8, it is found that when increasing the platform speed, the energy density of the noise spectrum is smaller, which leads to a larger SNR, and the detectability of the shorter waves ( $\mathrm{SNR}>1)$ is increased.

Results when changing the altitude of the platform from $2000 \mathrm{~m}$ to $5000 \mathrm{~m}$ are shown in Fig. 12. Increasing the flight height means a larger value of $L_{\phi}$ in (11), and hence a decrease in $P_{\text {mod }}$. This leads to a larger contribution of $N_{\text {surf }}$ and smaller contribution of $N_{\text {int }}$ with respect to our simulations of Fig. 7 and 8 , and explains why the sensitivity to with speed or wave height is different from the case of Fig. 7 to 8 .

Compared with Fig. 7 and Fig. 8, it can be seen that the omnidirectional energy of the noise spectrum is slightly smaller, in particular at small $K$. This is because the higher flight level results in a larger value of $L_{\phi}$ and hence of $N_{\text {int }}$ in (8f). On the other hand, $P_{\text {mod }}^{*}$ decrease with $L_{\phi}$. The combination of both effects finally results in a smaller SNR. Furthermore, the larger sensitivity to wind-sea wave height as compared to Fig. 7 is because, for a higher flight height while keeping all other parameters constant, $N_{\text {surf }}$ may become dominant in the speckle energy spectrum. Increasing the wind speed (and so the wind wave Hs) induces an increase in $m_{t t}$ and $N_{\text {surf }}$, which explains the decreasing trend of the density of the spectrum for increasing wind speed (Fig. 12(a)). Furthermore, as $m_{t t}$ is mainly determined by the wind waves and not very sensitivity to long swell, the impact of increasing Hs remains small in the swell dominated case (Fig.12(b)).

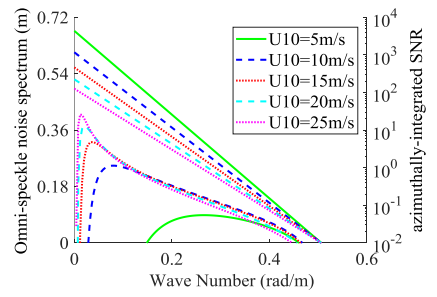

(a)

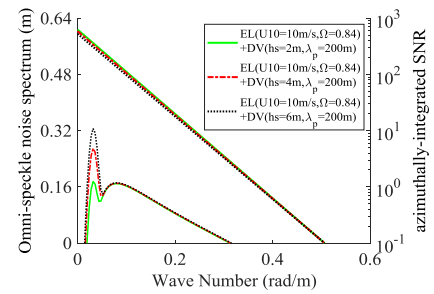

(b)
Fig. 12. Omni-directional speckle noise spectrum (left Y-axis) and SNR (right Y-axis) as a function of the wavenumber $\mathrm{K}$ for the flight height of $5000 \mathrm{~m}$, the sea surface conditions are the same as those of Fig. 7 and Fig. 8 for (a) and (b), respectively.

Next, we study the impact of the beam aperture in azimuth. Fig. 13 show the omni-directional speckle noise spectrum when the $3 \mathrm{~dB}$ beam width in azimuth $\beta_{\phi}$ is $17.2^{\circ}$, namely two times that given in Table I, other parameters are the same as those used for Fig.7 and Fig. 8, respectively. When $\beta_{\phi}$ increases, $N_{m o v}$ is larger according to (8c) and (5c), and $N_{\text {int }}$ is also larger according to (8f). The results show that the omni-directional energy of the noise spectra decreases. On the contrary, compared with Fig. 7 and Fig. 8, the SNR is not modified when $\beta_{\phi}$ is increased. It is because $\mathrm{SNR} \propto N_{\text {tot }} * P_{\text {mod }}$, where $N_{\text {tot }} \propto \beta_{\phi}, P_{\text {mod }} \propto\left(\beta_{\phi}\right)^{-1}$. Correlatively, in this case, similarly to the cases of Fig. 7 and 8 , the energy of the speckle noise spectrum does not vary significantly with the sea conditions. The results illustrated by Fig. 13 also show that in terms of SNR, it is not equivalent to increase the footprint dimension by flying higher or by increasing the beam aperture in azimuth. SNR is not affected by increasing the footprint, whereas increasing the flight height results in a decrease of the SNR.

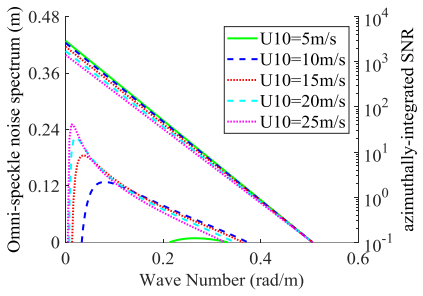

(a)

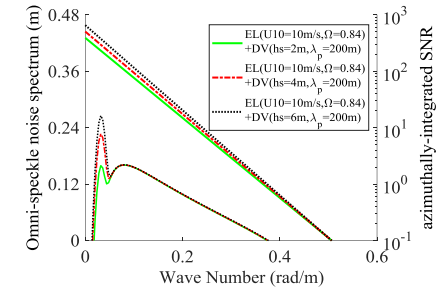

(b)
Fig. 13. Omni-directional speckle noise spectrum (left Y-axis) and SNR (right Y-axis) as a function of the wavenumber $\mathrm{K}$ for $3 \mathrm{~dB}$ beam width in azimuth $\beta_{\phi}=17.2^{\circ}$, the sea surface conditions are the same as those of Fig. 7 and Fig. 8 for (a) and (b), respectively.

Now let us discuss the effect of the radar frequency. Fig. 14(a) plots the omni-directional speckle noise spectrum in C-band $(5 \mathrm{GHz}), \mathrm{Ku}-$ band $(13.5 \mathrm{GHz})$ and $\mathrm{Ka}-$ band $(37.5 \mathrm{GHz})$ for case 1 of Table II (fully developed wind-waves with $U_{10}=10 \mathrm{~m} / \mathrm{s}$ ). The other radar parameters are the same as those in Table I. From (5c), (8e) and (11), it is obvious that the radar frequency affects $N_{\text {platf }}$ and $N_{\text {surf }}$ through the electromagnetic wave number $k$ and the $m_{t t}$ value, and effects $N_{\text {int }}$ through $k, m_{t t}$ and the tilting sensitivity term $\frac{\partial \ln \sigma^{0}}{\partial \theta}$. With the radar frequency, $k$ and $m_{t t}$ increase and $\frac{\partial \ln \sigma^{0}}{\partial \theta}$ decreases, which leads to the increases of $N_{\text {int }}, N_{\text {surf }}$ and $N_{\text {platf }}$, then the decrease of speckle spectrum decreases. On the other hand, the increase of the radar frequency also leads to the decrease of the modulation spectrum $P_{\text {mod }}$ through $\frac{\partial \ln \sigma^{0}}{\partial \theta}$, see (11). The overall effect on the speckle spectrum is that both the value at the origin $(K=0)$ and the absolute value of the slope with $K$ decrease when increasing the electromagnetic frequency ( $\mathrm{C}$ to Ka-band). The impact is important with a change by a factor of 2.9 on the value at the origin and on the linear slope. As for the SNR, Fig. 14(a) shows that peak value increases by a factor of 4.2 from C-band to Ka-band and the detectability of the shorter waves (wavenumber for which $\mathrm{SNR}>1$ ) significantly increases also. This shows that the overall impact of increasing the radar frequency is to increase the SNR, indicating that the lower values of $P_{\text {mod }}$ when increasing the radar frequency (due to a lower value of the tilt sensitivity term $\frac{\partial \ln \sigma^{0}}{\partial \theta}$ ) are over-balanced by the lower values of speckle energy. We can conclude from this, that for a same sea surface condition, same radar geometry and same radar bandwidth, a Ka-band configuration is better than a Ku or C-Band to minimize the speckle effect in the signal modulations.

Next, we study the effect of the radar incidence angle. Fig. 14(b) shows the omni-directional speckle noise spectrum at the 
incidence angle of $6^{\circ}, 10^{\circ}, 14^{\circ}$. The other radar parameters are the same as those in Table I and the sea surface conditions are the same as in case 1 (fully developed wind-waves with $U_{10}=$ $10 \mathrm{~m} / \mathrm{s})$. Both the value at the origin $(K=0)$ and the absolute value of the slope with $K$ decrease with the incidence angle. On the one hand, the incidence angle affects the speckle noise spectrum with the variable $K_{p}$, which is included both in the triangle function and in $N_{\text {tot }}$. From (5b), $K_{p}$ is proportional to the sine of the incidence angle. On the other hand, the incidence angle also affects $N_{\text {surf }}\left(N_{\text {surf }}\right.$ decreasing with incidence) and $N_{\text {int }}$ (opposing effects of $\frac{\partial \ln \sigma^{0}}{\partial \theta}$ and $m_{t t}$ ). The combination of these effects leads to what is shown as Fig. 14(b). When decreasing the incidence angle from $14^{\circ}$ to $6^{\circ}$, the density of the spectrum at $K=0$ increase by 2.1 and the absolute value of the slope decrease by a factor of 4.8. Correlatively, the SNR at the peak of the spectrum decreases by a factor of 4.7 .

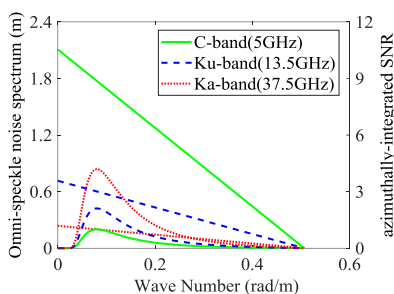

(a)

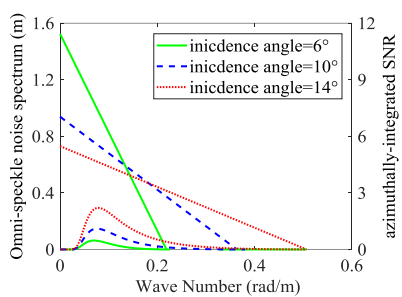

(b)
Fig. 14. Omni-directional speckle noise spectrum (left Y-axis) and SNR (right Y-axis) as a function of the wavenumber K, (a) for C, Ku and $\mathrm{Ka}$ band, (b) at the incidence angle of $6^{\circ}, 10^{\circ}$ and $14^{\circ}$ other radar parameters are the same as in Table I, the sea surface conditions are those of case 1 in Table II.

These latter tests indicate that the energy of the noise spectrum in the wave energy containing part decrease very significantly with the radar frequency and with the incidence angle. Correlatively, the SNR, which characterizes the detectability of the tilting waves in the fluctuation spectra, increase very significantly with the radar frequency and the incidence angle. Thus, in order to optimize the wave detection from the fluctuation spectra, while keeping the conditions of the measurement concept (tilting wave measurements in a GO approximation), the conditions are optimized for a high radar frequency and relatively large near-nadir incidence angles.

\section{CONCLUSION}

When ocean wave spectra are to be estimated from fluctuation spectra of the normalized backscattering coefficient of the sea surface, a correction must be applied to account for speckle noise effects in the measured fluctuation spectra or a special method must be implemented to eliminate the speckle noise effect. This assertion is true for observations from either SAR measurements or from real-aperture scanning radars. The speckle results from the interaction of the electromagnetic waves with the surface so that it is governed by both the instrumental configuration (in particular the velocity of the platform, the footprint dimension, and the azimuth angle) and the surface conditions. There are very few studies which propose a parametric model to describe the speckle noise behavior as a function of these parameters.

In this paper, we have proposed a modified version of the theoretical speckle model established by [1] and [11] for a nearnadir scanning geometry, by removing two limiting approximations. First, we have considered a moving instead of a frozen surface, in order to take into account the impact of surface scatter motions on the backscattered signal. Secondly, we have suppressed the approximation which consists in ignoring the four-frequency moment of the scattering matrix for wave number differences close to zero. We proposed a formulation where these two limits are suppressed while keeping the same condition of the scattering approximation (GO approximation). First, we simulated the speckle noise behavior for a $\mathrm{Ku}$ band radar with a mean incidence angle of $13^{\circ}$ (similar to that of the airborne radar KuROS) for different sea-state conditions and evaluated the different contributions to the speckle energy spectrum. We also evaluated a signal-to-noise ratio (SNR) in the spectral domain (SNR defined as the ratio of the spectral energy of signal fluctuations due to the long tilting waves to that due to speckle).

The simulation results show the important impact of the angle between flight and wave direction on the azimuthal variation of speckle and, for the chosen conditions (Table I) the relatively small impact of the sea-state conditions. Our simulation reveals that although the speckle energy is maximum in the along-track direction as expected from the simpler model of Jackson, its energy in this direction is smaller than that for a frozen surface model. When the radar antenna looks into the flight direction, the surface scatters motion explains the fact that the speckle level remains compatible with the inversion of wave properties in this direction in spite of the high speckle induced by the platform speed.

Besides, the angle between the flight direction and the wave propagation direction has an important impact on the variations of speckle energy with azimuth near the across-track direction, with the absence of a well-marked minimum in the across-track direction when waves propagate across-track. The minimum values of speckle in an azimuthally scanning geometry are dependent on the angle between radar look direction and flight direction, on the angle between flight direction and wave propagation direction, and on sea surface conditions.

The model was validated against empirical speckle values derived from observations with the $\mathrm{Ku}$-band airborne radar in 11 different cases (different flight legs with variable sea state conditions and different flight geometry with respect to the wave direction). The errors between the omni-directional noise spectrum from KuROS and from the model predictions all below $10 \%$ for all the cases. The detailed comparisons between model and observations show that only our model is able to reproduce the detailed behavior of the speckle variation with the radar look angle and the trend with wavenumber.

The new terms that we introduced to correct the Jackson's model are both necessary to guarantee the consistency of the model for the speckle noise spectrum. The term in $N_{\text {surf }}$, which is related to the velocity variance of scatters is necessary to explain the behavior of the speckle when the antenna looks in the flight direction and the trend of the speckle level with wind speed and wave age. The term in $N_{\text {int }}$ which mainly comes from the ratio of the modulation spectrum $P_{\text {mod }}$ and the velocity variance of scatters, is necessary to reproduce the variations of speckle with the radar azimuth angle, and with the angle between the flight and the wave propagation direction.

Once validated against observations, additional simulations have been carried to evaluate the sensitivity of the speckle to various radar configurations. The results show that:

$1)$. The effect of the sea surface conditions on the omnidirectional speckle noise spectrum depends on radar configurations. For the case of moderate flight speed (e.g.; 100 $\mathrm{m} / \mathrm{s}$ as the one corresponding to the KuROS data), the omnidirectional density spectrum is only slightly sensitive to sea state conditions (wind waves or swell). For higher speeds the density of the speckle noise spectrum becomes smaller, which induces an increase of SNR. In this high speed case, the noise spectrum increases with the significant height Hs of the sea surface (either wind sea or swell), and decreases with the wave length of the swell component. For a case of higher flight height, 
both the density of the speckle noise spectrum and SNR become smaller. In this case, the surface scatters velocity variance plays a more important role. When increasing the beam aperture in azimuth, the energy of the noise spectrum decreases, but the SNR does not change. In this case, the sensitivity of the speckle energy to wind speed and significant wave height is weak.

2). The energy of the speckle spectrum decreases with the radar frequency and the SNR increases. In particular our simulations show that using Ka-band we can obtain a SNR 2 times larger than in Ku-band (keeping all parameters identical). The effect of the radar incidence angle is obvious, with both the value at the origin $(K=0)$ and the slope of the trend with $K$ decreasing with the incidence angle.

To extrapolate the present results to the case of the satelliteborne scatterometer SWIM, the following remarks can be made. For both KuROS and SWIM conditions, $N_{\text {mov }}$ decreases sharply near the along-track direction, where the spectral density of speckle is mainly determined by the variance of sea surface vertical motion velocity $m_{t t}$. This probably explains the results presented in [3] where the speckle density spectrum in the along-track direction was found to be sensitive to the seasurface conditions (decreasing with wind speed as in the simulations with high flight height presented here above).

However, there are three main differences between SWIM and KuROS. Firstly, the satellite platform moves at a speed of $7 \mathrm{~km} / \mathrm{s}$, which results in $\mathrm{Bd}>>$ PRF for all angles far from the along-track direction. For these angles, the spectral density is mainly determined by the PRF, so that it is not impacted by the observation angle nor sea surface conditions. This is compatible with the results of [3] who could not evidence a dependence of speckle with sea-state for these look angles. The second difference is that the motion induced by the Earth rotation cannot be neglected in a satellite configuration case. This means that in order to apply our model to the SWIM-like configuration, this effect must be added. Thirdly, the height of the satellite platform is $520 \mathrm{~km}$, and the antenna azimuth gain parameter $L_{\Phi}$ is hundreds of times of KuROS, which leads to the contribution of $N_{i n t}$ much less than that of KuROS. Indeed, preliminary results indicate that in the worst case (i.e. for high sea-state conditions and wave propagation direction close to the along the flight direction), the contribution of the term in $N_{\text {int }}$ is $18 \%$, i.e. much less than what was shown in Fig. 6 .

In a future work we plan to use our model in the satelliteborne scatterometer SWIM configuration, first to compare with the empirical model established from the data (see [3]) and if necessary, to improve the speckle noise spectrum rejection included in the fluctuation spectrum measured by SWIM.

More generally, the formalism proposed in our model may also help to find optimal configurations for future satellite missions. For example, our results reveal that as concerns the wave modulation to speckle ratio (SNR) the configuration is more favorable in $\mathrm{Ka}$ band than in $\mathrm{Ku}$ Band. It is also more favorable at 10 to $12^{\circ}$ than at $6^{\circ}$. These results may help to choose the optimal configuration for a mission like SKIM [21].

\section{APPENDIX A: DERIVATION OF THE FLUCTUATION SPECTRUM}

First note that the geometry of observation is given in Fig. A1 and the list of mathematical symbols is given in Appendix B.

We start with the scattering function which appears in (4), but we express it from now on in the wavenumber domain (instead of frequency domain) and note it $\mathrm{S}(k, t)$. To express $\mathrm{S}(k, t)$ we use, similarly to [1], the physical optics integral solution of the surface scattering transfer function by [23]:
$S(k, t)=\frac{i k \sec \theta}{2 \pi r_{0}} \int G(\vec{x}-\vec{V} t) \exp [-i 2 k r(\vec{x}, t)] d \vec{x}$

where the reference frame is taken at $t=0$ and its origin is located at the center of the beam spot, $\theta$ is the incidence angle, $G(\vec{x})$ is the antenna gain pattern projected on the horizon plane, $\vec{V}$ is the displacement velocity vector of the radar which is supposed to be of no z-axis component, i.e. $\vec{V} \cdot \overrightarrow{e_{z}}=0 . d \vec{x}=$ $d x_{1} d x_{2}, \vec{x}=x_{1} \vec{\rho}+x_{2} \vec{\phi}$ with $\vec{\rho}$ being the unit vector along the horizontal look direction of the radar, $\vec{\phi}$ being unit vector perpendicular to $\vec{\rho}$ in the horizontal plane.

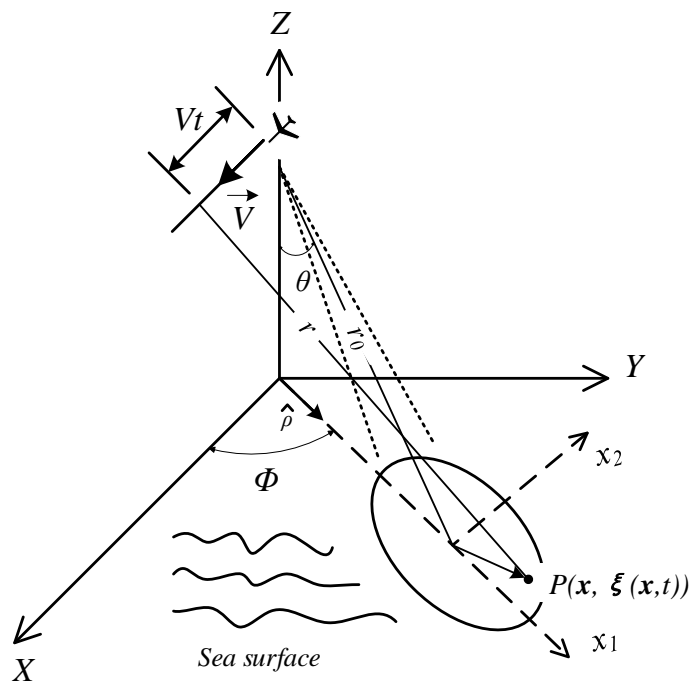

Fig. A1. the diagram of radar geometry

As shown in fig. A1, the range vector from the radar to the ocean surface point $\mathrm{P}$ is $\vec{r}=(\vec{x}, \xi(\vec{x}, t))-\overrightarrow{r_{0}}-\vec{V} t$, where $\overrightarrow{r_{0}}$ denotes the position vector from the beam spot center to the radar at $t=0$, and where $\xi(\vec{x}, t)$ is the instantaneous elevation of the surface.

The four-frequency-moment in (4) is re-expressed in the wavenumber domain:

$$
M\left(k, k^{\prime}, \kappa, \Delta t\right)=<S(k, t) \cdot S^{*}(k-\kappa, t) \cdot S^{*}\left(k^{\prime}, t+\Delta t\right) \cdot
$$$$
S\left(k^{\prime}-\kappa, t+\Delta t\right)>
$$

$k, k^{\prime}$ is the wave number of the transmitted pulse, $\kappa$ is the wave number difference of the transmitted pulse, $\Delta t$ is the time interval.

For a usual radar detection system, such as KuROS and SWIM, the Fresnel approximation can be assumed since $\left|\overrightarrow{r_{0}}\right| \gg$ $|(\vec{x}, \xi(\vec{x}, t))-\vec{V} t|$. Therefore, we expand the magnitude of $\vec{r}$ to the second order, then substitute $\mathrm{r}$ into (A1), according to (A2), the four-frequency moment becomes:

$$
\begin{aligned}
M\left(k, k^{\prime}, \kappa, \Delta t\right)= & \left(\frac{k \sec \theta}{2 \pi r_{0}}\right)^{4} \iiint \int G\left(\overrightarrow{x_{1}}\right) G\left(\overrightarrow{x_{2}}\right) \\
& G\left(\overrightarrow{x_{3}}-\vec{V} \Delta t\right) G\left(\overrightarrow{x_{4}}-\vec{V} \Delta t\right) \\
& <\exp \left\{i 2 \operatorname { c o s } \theta \left[k\left(\xi_{1}-\xi_{2}-\xi_{3, \Delta t}+\xi_{4, \Delta t}\right)+\right.\right. \\
& \left.\left.\Delta k\left(\xi_{3, \Delta t}-\xi_{4, \Delta t}\right)+\kappa\left(\xi_{2}-\xi_{4, \Delta t}\right)\right]\right\}> \\
& \exp \left\{i 2 \operatorname { s i n } \theta \vec { \rho } \cdot \left[k\left(\overrightarrow{x_{1}}-\overrightarrow{x_{2}}-\overrightarrow{x_{3}}+\overrightarrow{x_{4}}\right)+\right.\right. \\
& \left.\Delta k\left(\overrightarrow{x_{3}}-\overrightarrow{x_{4}}\right)+\kappa\left(\overrightarrow{x_{2}}-\overrightarrow{x_{4}}\right)+\kappa \vec{V} \Delta t\right]+ \\
& \varphi\} d \overrightarrow{x_{1}} d \overrightarrow{x_{2}} d \overrightarrow{x_{3}} d \overrightarrow{x_{4}}
\end{aligned}
$$

where $\xi_{i}$ represents the height of the sea surface at the time $t$, $\xi_{i, \Delta t}$ represents the height of the sea surface at the time of $t+$ $\Delta t, i . e ., \xi_{i}$ denotes $\xi\left(\overrightarrow{x_{l}}, t\right)$, and $\xi_{i, \Delta t}$ denotes $\xi\left(\overrightarrow{x_{l}}, t+\Delta t\right), \varphi$ is a phase related to the second order term of $\vec{r}$.

$$
\begin{aligned}
\varphi=- & \frac{i}{r_{0}}\left[k\left(\left|\overrightarrow{x_{1}}\right|^{2}-\sin ^{2} \theta\left|\vec{\rho} \cdot \overrightarrow{x_{1}}\right|^{2}\right)-(k-\kappa)\left(\left|\overrightarrow{x_{2}}\right|^{2}-\right.\right. \\
& \left.\sin ^{2} \theta\left|\vec{\rho} \cdot \overrightarrow{x_{2}}\right|^{2}\right)-k^{\prime}\left(\left|\overrightarrow{x_{3}}-\vec{V} \Delta t\right|^{2}-\sin ^{2} \theta \mid \vec{\rho} \cdot\left(\overrightarrow{x_{3}}-\right.\right.
\end{aligned}
$$




$$
\begin{aligned}
& \left.\vec{V} \Delta t)\left.\right|^{2}\right)+\left(k^{\prime}-\kappa\right)\left(\left|\overrightarrow{x_{4}}-\vec{V} \Delta t\right|^{2}-\sin ^{2} \theta \mid \vec{\rho} \cdot\left(\overrightarrow{x_{4}}-\right.\right. \\
& \left.\left.\vec{V} \Delta t)\left.\right|^{2}\right)\right]
\end{aligned}
$$

If let $\Delta t=0$ and ignore the height change of the sea surface with the time $t$, then the expression of (A3) is the same as (17) of [1].

For large $k$, the dominant contributions to the moment come only from the neighborhood of two stationary points, i.e. $\mathrm{D} 1: \overrightarrow{x_{1}}=\overrightarrow{x_{2}}, \overrightarrow{x_{3}}=\overrightarrow{x_{4}}$ or $\mathrm{D} 2: \overrightarrow{x_{1}}=\overrightarrow{x_{3}}, \overrightarrow{x_{2}}=\overrightarrow{x_{4}}$. The sets D1 and D2 are distinct and yield distinct contributions to the moment; i.e., $M=M_{1}+M_{2}$, where $M_{1}$ and $M_{2}$, respectively, derive from integrations over the small volumes surrounding D1 and D2.

In the following, we derive separately the four-frequency moments $M_{1}$ and $M_{2}$, and obtain the fluctuation spectra $\widetilde{\mathrm{P}}_{1}$ and $\widetilde{\mathrm{P}}_{2}$ for the cases of D1 and D2, respectively. The total fluctuation spectrum $\widetilde{P}=\widetilde{\mathrm{P}}_{1}+\widetilde{\mathrm{P}}_{2}$. We will see that $\widetilde{\mathrm{P}}_{1}$ represents the fluctuation spectrum in absence of fading (speckle) noise, whereas $\widetilde{P}_{2}$ is the speckle contribution.

In the actual data processing, we usually use the relative fluctuation spectra $\mathrm{P}$ of backscattering coefficient, which is defined as a fluctuation spectrum normalized by the variance of backscattering coefficient.

$$
\begin{aligned}
P=\frac{\tilde{P}}{\left\langle\left|e_{S}(\tau, t)\right|^{2}\right\rangle^{2}} & \\
\text { with }\left\langle\left|e_{S}(\tau, t)\right|^{2}>\right. & =<\int E_{0}(k) E_{0}^{*}(k) S(k) S^{*}(k) d v> \\
= & {\left[<|S(k)|^{2}>\right]^{2}(2 \pi B)^{2} }
\end{aligned}
$$

where $E_{0}(\mathrm{k})$ is the Fourier Transform (FT) of the incident shortpulsed waveform, B is the bandwidth of the transmitted pulse.

In the following, the derivation is carried out by assuming a Gaussian pattern for the antenna gain

$$
G(\vec{x})=\exp \left(-\frac{x_{1}^{2}}{2 L_{\rho}^{2}}-\frac{x_{2}^{2}}{2 L_{\phi}^{2}}\right)
$$

\section{A1. FLUCTUATION SPECTRUM FOR THE D1 DOMAIN AND ITS RELATION WITH THE WAVE SLOPE SPECTRUM}

The approach is similar to the development described in [1], except that time is considered as an additional variable of the sea surface elevation:

We first define the set of variables:

$\vec{u}=2 k \cos \theta\left(\overrightarrow{x_{1}}-\overrightarrow{x_{2}}\right), \vec{v}=2 k \cos \theta\left(\overrightarrow{x_{3}}-\overrightarrow{x_{4}}\right), \vec{w}=\overrightarrow{x_{2}}-\overrightarrow{x_{4}}$, $\vec{x}=\overrightarrow{x_{4}} \quad$ (A6)

Then we expand the height differences in the neighborhoods of the stationary points D1 in a Taylor series to the first order in space interval $\vec{u}, \vec{v}$ and time interval $\Delta t$.

$2 k \cos \theta\left(\xi_{1}-\xi_{2}\right)=\nabla \xi_{2} \cdot \vec{u}$,

$2 k \cos \theta\left(\xi_{3, \Delta t}-\xi_{4, \Delta t}\right)=\nabla \xi_{4, \Delta t} \cdot \vec{v}, \xi_{i, \Delta t}=\xi_{i}+\Delta t \frac{\partial \xi_{i}}{\partial t}$

Where $\nabla \triangleq\left(\frac{\partial}{\partial x_{1}}, \frac{\partial}{\partial x_{2}}\right)$.

Then the four-frequency moment in the D1 domain can be expressed as:

$$
\begin{aligned}
M_{1}(\vec{K}, \Delta \mathrm{t})= & \left(\frac{\sec ^{2} \theta}{2 r_{0}}\right)^{4} \int B(\vec{w}, \Delta t) \Xi(\vec{w}, \Delta t ; \vec{K}) \\
& \exp [-i \vec{K}(\vec{w}+\vec{V} \Delta t)] d \vec{w}
\end{aligned}
$$

where $\vec{K}=2 \kappa \sin \theta \vec{\rho}$

$$
\begin{gathered}
B(\vec{w}, \Delta t)=\int G^{2}(\vec{x}+\vec{w}) G^{2}(\vec{x}-\vec{V} \Delta t) \exp \left(\varphi_{1}\right) d \vec{x} \\
\varphi_{1}=\left\{-\frac{i \kappa}{r_{0}}\left[\cos ^{2} \theta\left(\left(x_{1}+w_{1}\right)^{2}-\left(x_{1}-V_{1} \Delta t\right)^{2}\right)\right]+\right. \\
\left.\left(x_{2}+w_{2}\right)^{2}-\left(x_{2}-V_{2} \Delta t\right)^{2}\right\}
\end{gathered}
$$

Where the subscript 1, 2 represents vector's component along $\vec{\rho}$ axis or $\vec{\phi}$ axis, respectively. $\varphi_{1}$ is the form of the phase term
(A3) for the case of D1.

Using the Gaussian assumption for the beam pattern, then (A9) becomes:

$B(\vec{w}, \Delta t)=\frac{\pi L_{\rho} L_{\Phi}}{2} \exp \left[-\frac{1}{2} b_{\alpha}^{2}\left(w_{\alpha}+V_{\alpha} \Delta t\right)^{2}\right]$

with $\alpha=1$ or 2 , corresponding to vector's component along $\vec{\rho}$ axis or $\vec{\phi}$ axis, respectively

$b_{1}^{2}=L_{\rho}^{-2}+\left(\frac{K L_{\rho}}{2 r_{0} \sin \theta}\right)^{2} \cos ^{4} \theta, b_{2}^{2}=L_{\Phi}^{-2}+\left(\frac{K L_{\Phi}}{2 r_{0} \sin \theta}\right)^{2}$

In (A7), $\Xi(\vec{w}, \Delta t ; \vec{K})$ is:

$\Xi(\vec{w}, \Delta t ; \vec{K})=\frac{1}{(2 \pi)^{4}} \iint<\cdots>\exp [-i \vec{s} \cdot(\vec{u}-\vec{v})] d \vec{u} d \vec{v}$

where $\vec{s}=\tan \theta \vec{\rho}$ is the local slope at the surface.

Bracket term $\langle\cdots\rangle$ in (A10) is the same as the bracket term in (A3). According to Longuet-Higgins's method [15], the exponential term can be re-expressed as a series of cumulants to the second order. Supposing the average of the sea surface elevation to be 0 , and applying the method presented in Papoulis [1965], the bracket term becomes:

$<\cdots>=\exp \left(-\frac{1}{2} \mu_{i j} l_{i} l_{j}\right)$

where the Einstein summation convention applies to repeated indices,

$\vec{l}=(\vec{u},-\vec{v}, 2 \kappa \cos \theta,-2 \kappa \cos \theta)$

and $\mu_{i j}$ is the following symmetric matrix.

$\mu_{i j}=<\overline{\bar{h}}>=$

$$
\left(\begin{array}{cccc}
m_{\alpha \beta} & -R_{\alpha \beta}+\Delta t R_{\alpha \beta \Delta t} & 0 & R_{\alpha}-\Delta t R_{\alpha \Delta t} \\
& m_{\alpha \beta} & -R_{\alpha}+\Delta t R_{\alpha \Delta t} & 0 \\
& & \sigma^{2} & R-\Delta t R_{\Delta t} \\
& & & \sigma^{2}
\end{array}\right)
$$

$\overline{\bar{h}}$ means dyad of vector $\vec{h}: \overline{\bar{h}}=\vec{h} \vec{h}$, with

$\vec{h}=\left(\nabla \xi_{2}, \nabla \xi_{4, \Delta t}, \xi_{2}, \xi_{4, \Delta t}\right)$,

In (A11b) the terms of the matrix are all defined from the correlation function:

$R=R(\vec{w}, 0)=\left\langle\xi_{2} \xi_{4}\right\rangle=\langle\xi(\vec{x}+\vec{w}, t) \xi(\vec{x}, t)\rangle$

$R_{\alpha}=\frac{\partial R(\vec{w}, 0)}{\partial w_{\alpha}}$ (for $\alpha, \beta=1$ or 2$)$, where the subscript of $\mathrm{R}$

and w means partial differential with respect to $w_{1}, w_{2}$ or $\Delta t$.

$m_{\alpha \beta}=-R_{\alpha \beta}(\mathbf{0}, 0)$,

$\sigma^{2}=R(\mathbf{0}, 0)$.

The matrix $\mu_{i j}$ is a symmetric matrix which can be separated as the sum of non-diagonal matrix $\mu_{i j}^{o}$ and diagonal matrix $\mu_{i j}^{d}$. Expanding the exponential term containing $\mu_{i j}^{o}$, then with the identities of (38) in [1], the integration in $\Xi$ is calculated, and $\Xi$ becomes:

$$
\begin{aligned}
\Xi(\vec{w}, \Delta t ; \vec{K})= & p^{2}(\vec{s}) \exp \left(-K^{2} \cot ^{2} \theta \sigma^{2}\right) \\
& {\left[1+K^{2} \cot ^{2} \theta\left(R-\Delta t R_{\Delta t}\right)+\right.} \\
& 2 i K \cot \theta \frac{P_{\alpha}}{P}\left(R_{\alpha}-\Delta t R_{\alpha \Delta t}\right)-\frac{p_{\alpha} p_{\beta}}{p^{2}}\left(R_{\alpha \beta}-\right. \\
& \left.\left.\Delta t R_{\alpha \beta \Delta t}\right)+\cdots\right]
\end{aligned}
$$

where $p(\vec{s})$ is the probability density function (pdf) of the sea surface slopes $\vec{s}$.

Substituting the four-frequency moment of $S(k, t)$ around D1 into (3), the Fourier transform of M1 is:

$$
\begin{aligned}
N_{1}\left(\vec{K}, \Omega_{0}\right)= & \frac{1}{2 \pi}\left(\frac{\sec ^{2} \theta}{2 r_{0}}\right)^{4} \iint B_{e}(\vec{w}, \Delta t) \Xi(\vec{w}, \Delta t ; \vec{K}) \\
& \exp (-i \vec{K} \cdot \vec{w}) d \vec{w} d \Delta t \\
B_{e}(\vec{w}, \Delta t)= & \frac{\pi L_{\rho} L_{\Phi}}{2} \exp \left[-\gamma^{2} \frac{\left(\Delta t+\frac{\vec{e} \cdot \vec{v}-i \Omega^{*}}{\gamma^{2}}\right)^{2}}{2}-\frac{\left(\Omega^{*}+i \vec{e} \cdot \vec{\gamma}\right)^{2}}{2 \gamma^{2}}-\frac{1}{2} \varrho^{2}\right]
\end{aligned}
$$


(A14)

Where $\Omega^{*}=\Omega_{0}-\vec{K} \cdot \vec{V}, \vec{\varrho}=\left(b_{1} w_{1}, b_{2} w_{2}\right)$, and $\vec{\gamma}=$ $\left(b_{1} V_{1}, b_{2} V_{2}\right)$. Because the bandwidth $\Omega_{0}$ of $W\left(\Omega_{0}-\vec{K} \cdot \vec{V}\right)$ is much larger than that of $N_{1}\left(\vec{K}, \Omega_{0}\right), W$ can be considered as a constant in $\Omega_{0}$ (let $\Omega_{0}=0$ ) and extracted outside of the integration, the integration on $\Omega_{0}$ in (1) could be calculated as: $\int W\left(\Omega_{0}-\vec{K} \cdot \vec{V}\right) N_{1}\left(\vec{K}, \Omega_{0}\right) d \Omega_{0}=W(0) \int N_{1}\left(\vec{K}, \Omega_{0}\right) d \Omega_{0}$

It is easy to prove that

$\int N_{1}\left(\vec{K}, \Omega_{0}\right) d \Omega_{0}=M_{1}(\vec{K}, \Delta \mathrm{t}=0)$

Finally, substituting (A15) to (1), and using a sinus cardinal shape for $W, W\left(\Omega_{0}\right)=\left[\frac{\sin \left(\Omega_{0} \cdot \frac{T_{i n t}}{2}\right)}{\Omega_{0} \cdot \frac{T_{i n t}}{2}}\right]^{2}$, the normalized fluctuation spectrum around D1 can be written as:

$P_{1}(K, \Phi)=\left[\operatorname{tri}\left(\frac{K}{2 \pi K_{p}}\right)\right]^{2} P_{\text {mod }}(K, \Phi)$

where

$$
\begin{aligned}
P_{\text {mod }}(K, \Phi)= & \frac{\sqrt{2 \pi}}{L_{\Phi}} \exp \left(-K^{2} \cot ^{2} \theta \sigma^{2}\right) \times \\
& {\left[\left(\cot ^{2} \theta-2 \cot \theta \frac{\mathrm{p}_{\alpha}}{\mathrm{p}} \frac{K_{\alpha}}{K}+\right.\right.} \\
& \left.\left.\frac{\mathrm{p}_{\alpha} \mathrm{p}_{\beta}}{p^{2}} \frac{K_{\alpha} K_{\beta}}{K^{2}}\right) K^{2} F(K, \Phi)+\cdots\right]
\end{aligned}
$$

(A17) and (A18) show that $P_{1}(K, \Phi)$ is proportional to the wave slope spectrum $K^{2} F(K, \Phi)$ for a given radar horizontal resolution $\delta x=1 / \mathrm{K}_{\mathrm{p}}$, thus $P_{1}(K, \Phi)$ is considered as the useful-signal-related part in total fluctuation spectrum, directly related to the waves to be measured.

(A18) is strictly equivalent to (47) of [1]. For the case of D1, $\langle\cdots\rangle$ is only related to the variation of the sea surface height with the spatial position in $M_{1}$ (see (A11)), and is not affected by the change of height with time. This explains the equivalence of (A18) and (47) of [1], although we considered here the more general case of a moving surface.

\section{A2. FLUCTUATION SPECTRUM FOR D2-SPECKLE NOISE SPECTRUM}

For four-frequency moment around the set of points D2, new series of variables are set:

$\overrightarrow{u^{\prime}}=2 k \cos \theta\left(\overrightarrow{x_{1}}-\overrightarrow{x_{3}}\right), \overrightarrow{v^{\prime}}=2 k \cos \theta\left(\overrightarrow{x_{2}}-\overrightarrow{x_{4}}\right), \overrightarrow{w^{\prime}}=\overrightarrow{x_{3}}-\overrightarrow{x_{4}}$, $\vec{x}=\overrightarrow{x_{4}}$

Similar to the case of D1, we express the height differences in the neighborhoods of the stationary points D2 by a Taylor series to the first order in space interval $\overrightarrow{u^{\prime}}, \overrightarrow{v^{\prime}}$ and time interval $\Delta t$.

$$
\begin{aligned}
& 2 k \cos \theta\left(\xi_{1}-\xi_{3, \Delta t}\right)=\nabla \xi_{3} \cdot \overrightarrow{u^{\prime}}-\Delta t \frac{\partial \xi_{3}}{\partial t}, \\
& 2 k \cos \theta\left(\xi_{2}-\xi_{4, \Delta t}\right)=\nabla \xi_{4} \cdot \overrightarrow{v^{\prime}}-\Delta t \frac{\partial \xi_{4}}{\partial t}
\end{aligned}
$$

Changing the integral element in four-frequency moment to the above variables, $M_{2}$ becomes:

$$
\begin{gathered}
M_{2}(\Delta K, \Delta t)=\left(\frac{\sec ^{2} \theta}{2 r_{0}}\right)^{4} \int B^{\prime}\left(\overrightarrow{w^{\prime}}, \Delta t\right) \Xi^{\prime}\left(\overrightarrow{w^{\prime}}, \Delta t ; \Delta K\right) \\
\quad \exp \left[-i \Delta K \vec{\rho} \cdot \overrightarrow{w^{\prime}}-\vec{K} \cdot \vec{V} \Delta t+\varphi_{2}\right] d \overrightarrow{w^{\prime}} \quad(\mathrm{A} 20) \\
\begin{aligned}
& B^{\prime}\left(\overrightarrow{w^{\prime}}, \Delta t\right)= G\left(\vec{x}+\vec{w}^{\prime}\right) G(\vec{x}) G\left(\vec{x}+\overrightarrow{w^{\prime}}-\vec{V} \Delta t\right) G(\vec{x} \\
&\quad-\vec{V} \Delta t) d \vec{x} \\
&= \frac{\pi L_{\rho} L_{\phi}}{2} \exp \left(-\frac{w_{1}^{2}+V_{1}^{2} \Delta t^{2}}{2 L_{\rho}^{2}}-\frac{w_{2}^{2}+V_{2}^{2} \Delta t^{2}}{2 L_{\Phi}^{2}}\right) \quad(\mathrm{A} 21) \\
&\left.\Xi^{\prime}\left(\overrightarrow{w^{\prime}}, \Delta t ; \Delta K\right)=\frac{1}{(2 \pi)^{4}} \iint<\cdots>\exp \left[-i \vec{s} \cdot \overrightarrow{u^{\prime}}-\overrightarrow{v^{\prime}}\right)\right] d \overrightarrow{u^{\prime}} d \overrightarrow{v^{\prime}}
\end{aligned}
\end{gathered}
$$

$\varphi_{2}=-\frac{i}{r_{0}}\left\{k\left[\cos ^{2} \theta\left(2 x_{1}+w_{1}\right) w_{1}+\left(2 x_{2}+w_{2}\right) w_{2}\right]+\right.$ $\kappa\left(\cos ^{2} \theta x_{1}^{2}+x_{2}^{2}\right)-k^{\prime}\left[\cos ^{2} \theta\left(2 x_{1}+w_{1}-\right.\right.$ $\left.\left.2 V_{1} \Delta t\right) w_{1}+\left(2 x_{2}+w_{2}-2 V_{2} \Delta t\right) w_{2}\right]-\kappa\left[\cos ^{2} \theta\left(x_{1}-\right.\right.$ $\left.\left.\left.V_{1} \Delta t\right)^{2}+\left(x_{2}-V_{2} \Delta t\right)^{2}\right]\right\}$

$\varphi_{2}$ is the form of the phase term (A3a) for the case of D2. Bracket term $\langle\cdots\rangle$ in (A22) is the same as the bracket term in (A3). As done for D1, we apply Longuet-Higgins' method and Papoulis' method to expand the bracket term and turn it into the distribution of cumulants. Then the bracket term becomes:

$<\cdots>=\exp \left(-\frac{1}{2} \mu_{i j}^{\prime} l_{i}^{\prime} l_{j}^{\prime}\right)$

where $\mu_{i j}^{\prime}$ is the following symmetric matrix.

$$
\left(\begin{array}{cccccc}
m_{\alpha \beta} & & & \multicolumn{3}{c}{\mu_{i j}^{\prime}=<\overline{\overline{h^{\prime}}}>=} \\
& -R_{\alpha \Delta t}(\mathbf{0}, 0) & -R_{\alpha \beta} & -R_{\alpha \Delta t} & 0 & R_{\alpha} \\
& m_{t t} & -R_{\alpha \Delta t} & -R_{\Delta t \Delta t} & 0 & R_{\Delta t} \\
& m_{\alpha \beta} & -R_{\alpha \Delta t}(\mathbf{0}, 0) & -R_{\alpha} & 0 \\
& & m_{t t} & -R_{\Delta t} & 0 \\
& & & & \sigma^{2} & R \\
& & & & & \sigma^{2}
\end{array}\right)
$$

with

$R=R(\vec{w}, 0)$

$\overrightarrow{l^{\prime}}=\left(\overrightarrow{u^{\prime}},-2 k^{\prime} \cos \theta \Delta t,-\overrightarrow{v^{\prime}}, 2 k^{\prime} \cos \theta \Delta t, 2 \Delta k \cos \theta\right.$, $-2 \Delta k \cos \theta)$

$\overrightarrow{h^{\prime}}=\left(\nabla \xi_{3}, \frac{\partial \xi_{3}}{\partial t}, \nabla \xi_{4}, \frac{\partial \xi_{4}}{\partial t}, \xi_{3}, \xi_{4}\right)$

$m_{t t}=-\partial^{2} R(\mathbf{0}, 0) / \partial \Delta t^{2}$

Expanding the non-diagonal matrix in exponential bracket term, $\Xi^{\prime}$ is obtained by integration as:

$\Xi^{\prime}\left(\overrightarrow{w^{\prime}}, \Delta t ; \Delta K\right)=p^{2}(\vec{s}) \exp \left(-4 k^{\prime} \cos ^{2} \theta m_{t t} \Delta t^{2}-\right.$

$$
\begin{aligned}
& \left.\Delta K^{2} \cot ^{2} \theta \sigma^{2}\right)\left[1-4 k^{\prime} \cos ^{2} \theta \Delta t^{2} R_{\Delta t \Delta t}+\right. \\
& \Delta K^{2} \cot ^{2} \theta R+2 i \Delta K \cot \theta \frac{p_{\alpha}}{p} R_{\alpha}- \\
& \left.\frac{p_{\alpha} p_{\beta}}{p^{2}} R_{\alpha \beta}+\cdots\right]
\end{aligned}
$$

To deal with the new function $\Xi^{\prime}\left(\overrightarrow{w^{\prime}}, \Delta t ; \Delta K\right)$, we write $\Xi^{\prime}$ as:

$$
\begin{aligned}
& \Xi^{\prime}=\Xi_{a}^{\prime}+\Xi_{b}^{\prime}, \\
& \Xi_{a}^{\prime}\left(\overrightarrow{w^{\prime}}, \Delta t ; \Delta K\right)= \mathrm{p}^{2}(\vec{s}) \exp \left(-4 \mathrm{k}^{\prime 2} \cos ^{2} \theta \mathrm{m}_{\mathrm{tt}} \Delta \mathrm{t}^{2}-\right. \\
&\left.\Delta K^{2} \cot ^{2} \theta \sigma^{2}\right) \\
& \Xi_{b}^{\prime}\left(\overrightarrow{w^{\prime}}, \Delta t ; \Delta K\right)= \mathrm{p}^{2}(\vec{s}) \exp \left(-4 \mathrm{k}^{\prime 2} \cos ^{2} \theta \mathrm{m}_{\mathrm{tt}} \Delta \mathrm{t}^{2}-\right. \\
&\left.\Delta K^{2} \cot ^{2} \theta \sigma^{2}\right) \times\left[-4 \mathrm{k}^{\prime 2} \cos ^{2} \theta \Delta \mathrm{t}^{2} \mathrm{R}_{\Delta \mathrm{t} \Delta \mathrm{t}}+\right. \\
& \Delta \mathrm{K}^{2} \cot ^{2} \theta R+2 \mathrm{i} \Delta \mathrm{Kcot} \theta \frac{\mathrm{p}_{\alpha}}{\mathrm{p}} R_{\alpha}- \\
&\left.\frac{\mathrm{p}_{\alpha} \mathrm{p}_{\beta}}{p^{2}} R_{\alpha \beta}+\cdots\right]
\end{aligned}
$$

To compare with Jackson's derived results more conveniently, first, let's restrict to the case $\Delta K \rightarrow 0$ in $\Xi_{a}^{\prime}$. Then the $\Xi_{a}^{\prime}$ becomes:

$\Xi_{a}^{\prime}\left(\overrightarrow{w^{\prime}}, \Delta t ; 0\right)=p^{2}(\vec{s}) \exp \left(-4 k^{\prime} \cos ^{2} \theta m_{t t} \Delta t^{2}\right)$

As $L_{\rho} \gg V_{1} \Delta t$ and $L_{\Phi} \gg V_{2} \Delta t, V_{1} \Delta t$ and $V_{2} \Delta t$ in (A21) can be ignored. Thus, by integrating $\overrightarrow{w^{\prime}}$ in (A21), and substituting $M_{2}(\Delta K, \Delta t)$ in (3), the Fourier transform of the four-frequency moment at the limit $\Delta K \rightarrow 0$ is:

$$
\begin{aligned}
N_{2 a, \Delta K \rightarrow 0}\left(\Delta K, \Omega_{0}\right)= & \frac{\left[<|S(k)|^{2}>\right]^{2}}{\sqrt{2 \pi} \beta_{d}^{\prime}} . \\
& \exp \left[-\frac{\Omega^{* 2}}{2 \beta_{d}^{\prime 2}}-\frac{1}{2}\left(q^{\prime} L_{\rho} \Delta K\right)^{2}-\frac{i \epsilon \Omega^{*}}{\beta_{d}^{\prime 2}}\right]
\end{aligned}
$$

$\beta_{d}^{\prime 2}=\beta_{d}^{2}+8 k^{2} \cos ^{2} \theta m_{t t}$

$q^{\prime 2}=\frac{\left(\frac{2 k V}{r_{0}}\right)^{2} L_{\Phi}^{2} \sin ^{2} \Phi+8 k^{2} \cos ^{2} \theta m_{t t}}{\beta_{d}^{\prime 2}}$ 
$\beta_{d}^{2}=\left(\frac{2 k V}{r_{0}}\right)^{2}\left(L_{\rho}^{2} \cos ^{4} \theta \cos ^{2} \Phi+L_{\phi}^{2} \sin ^{2} \Phi\right)$

$\epsilon=\frac{2 k V}{r_{0}} L_{\rho}^{2} \cos ^{2} \theta \cos \Phi \Delta K$

With $\quad \Delta K \rightarrow 0$, (meaning $\Delta K \ll k$ ), the corresponding fluctuation spectrum before normalization can be calculated as:

$$
\begin{gathered}
P_{2 a, \Delta K \rightarrow 0}(\omega)=c \int_{c}\left|E_{0}(k c)\right|^{2}\left|E_{0}(k c-K c / 2 \sin \theta)\right|^{2} d k \\
\frac{c}{2 \sin \theta} \iint_{-\delta \Delta K}^{+\delta \Delta K} W\left(\Omega_{0}-\vec{K} \cdot \vec{V}\right) \\
\cdot N_{2, \Delta K \rightarrow 0}\left(\Delta K, \Omega_{0}\right) d \Delta K d \Omega_{0}
\end{gathered}
$$

Because $\mathrm{N}_{2 a, \Delta \mathrm{K} \rightarrow 0}\left(\Delta K, \Omega_{0}\right)$ is a Gaussian function of $\Delta K$ (see A29), then

$$
\begin{aligned}
& \int_{-\delta \Delta K}^{+\delta \Delta K} \mathrm{~N}_{2 a, \Delta \mathrm{K} \rightarrow 0}\left(\Delta K, \Omega_{0}\right) d \Delta K \\
& =\frac{\sqrt{2 \pi}}{T_{r}}\left[<|S(k)|^{2}>\right]^{2} \cdot \frac{\exp \left(-\Omega^{* 2} / 2 q^{\prime 2} \beta_{d}^{\prime 2}\right)}{\sqrt{2 \pi} q^{\prime} \beta_{d}^{\prime}}
\end{aligned}
$$

Since the $\Omega_{0}$ bandwidth of (A30) is much lower than that of $W,(\mathrm{~A} 30)$ can be considered as a constant (let $\Omega_{0}=0$ ) and extracted outside of the integration of $\Omega_{0}$.

Furthermore, using (A4) and a sinus cardinal shape of width $B$ for $W$ the corresponding normalized fluctuation spectrum is obtained:

$P_{2 a, \Delta K \rightarrow 0}(K)=\frac{\sqrt{2 \pi}}{L_{\rho}} \operatorname{tri}\left(\frac{K}{2 \pi K_{p}}\right) \frac{1}{2 \pi K_{p}} \frac{1}{q^{\prime} \beta_{d}^{\prime} T_{\text {int }} / \sqrt{2 \pi}}$

Where $q^{\prime} \beta_{d}^{\prime} / \sqrt{2 \pi}$ is a Doppler bandwidth which includes contributions from both the displacement of the platform and the movement of the sea surface. $q^{\prime} \beta_{d}^{\prime}$ is similar to $q \beta_{d}$ in (72) of [1], except that the Doppler bandwidth now includes the contribution due to the velocity of scatters.

As for the case where $\Delta K \neq 0$ in $\Xi_{a}^{\prime}, \Xi_{a}^{\prime}$ becomes: $\Xi_{a}^{\prime}\left(\overrightarrow{w^{\prime}}, \Delta t ; \Delta K\right)=p^{2}(\vec{s}) \exp \left(-4 k^{\prime} \cos ^{2} \theta m_{t t} \Delta t^{2}-\right.$

$$
\left.\Delta K^{2} \cot ^{2} \theta \sigma^{2}\right)
$$

To simplify the derivation process, the phase term $\varphi_{2}$ in (A23) is ignored. Then after integration $N_{2 a, \Delta K \neq 0}\left(\Delta K, \Omega_{0}\right)$ is obtained:

$$
\begin{aligned}
N_{2 a, \Delta K \neq 0}\left(\Delta K, \Omega_{0}\right)= & \frac{1}{\sqrt{2 \pi} L \rho}\left[<|S(k)|^{2}>\right]^{2} \sqrt{\frac{\pi}{\gamma^{\prime 2}+\hat{\alpha}}} \\
& \exp \left(-\frac{\Omega^{* 2}}{4\left(\gamma^{\prime 2}+\widehat{\alpha}\right)}\right) \frac{L_{\rho}}{\sqrt{2 \pi}} \exp \left[-\frac{1}{2}\left(\Delta K L_{\rho}\right)^{2}-\right. \\
& \left.\Delta K^{2} \cot ^{2} \theta \sigma^{2}\right]
\end{aligned}
$$

$N_{2 a, \Delta K \neq 0}\left(\Delta K, \Omega_{0}\right)$ can be regarded as a Gaussian function about $\Delta K$, and its width is mainly around $\Delta K \rightarrow 0$. However, the integration area of the corresponding fluctuation spectrum of $N_{2 a, \Delta K \neq 0}\left(\Delta K, \Omega_{0}\right)$ only covers area where $\Delta K \neq 0$, thus the corresponding fluctuation spectrum of $N_{2 a, \Delta K \neq 0}\left(\Delta K, \Omega_{0}\right)$ is nearly 0 .

\section{$P_{2 a, \Delta K \neq 0}=0$}

Now, considering the part of $\Xi_{b}^{\prime}$ that has been ignored by [1], (A27b) needs to be completely preserved. To simplify the derivation process, the second order term $\varphi_{2}$ (A23) is ignored. Substituting (A27b) into (A20), and calculating the integration over $\overrightarrow{w^{\prime}}$, the four-frequency moment is obtained; then substituting the four-frequency moment into (3) and calculating the integration over $\Delta t, N_{2 b}$ can be written as:

$\left.N_{2 b}\left(\Delta K, \Omega_{0}\right)=\frac{1}{\sqrt{2 \pi} L_{\rho}}\left[<|S(k)|^{2}\right\rangle\right]^{2} \sqrt{\frac{\pi}{\gamma^{\prime 2}+\widehat{\alpha}}} \exp \left[-\frac{\Omega^{* 2}}{4\left(\gamma^{\prime 2}+\widehat{\alpha}\right)}\right]$

Here $\gamma^{\prime 2}=b_{1}^{\prime 2} V_{1}^{2}+b_{2}^{\prime 2} V_{2}^{2}, b_{1}^{\prime 2}=\frac{1}{2 L_{\rho}^{2}}, b_{2}^{\prime 2}=\frac{1}{2 L_{\Phi}^{2}}$,

$\hat{\alpha}=4 k^{2} \cos ^{2} \theta m_{t t}$.

Since $\hat{\alpha} \gg \gamma^{\prime 2}, \gamma^{\prime 2}$ is ignored in the future calculation. Substituting (A35) in (1), then the normalized fluctuation spectrum related to $\Xi_{b}^{\prime}$ can be calculated as:

$P_{2 b}(K)=\operatorname{tri}\left(\frac{K}{2 \pi K_{p}}\right) \frac{1}{2 \pi K_{p} \operatorname{Tint}} \sqrt{\frac{\pi}{\widehat{\alpha}}} \int \operatorname{Pmod}^{*}(\Delta K, \Phi) d \Delta K$

Where $\mathrm{P}_{\text {mod }}^{*}(\Delta K, \Phi)$ is given as (8h). Combining (A31), (A34) and (A36), we obtain the normalized fluctuation spectrum $P_{2}(K, \Phi)$ for D2, :

$$
\begin{aligned}
P_{2}(K, \Phi)= & \operatorname{tri}\left(\frac{K}{2 \pi K_{p}}\right) \frac{1}{2 \pi K_{p}} \\
& \cdot\left(\frac{1}{q^{\prime} \beta_{d}^{\prime} T_{\text {int }} / \sqrt{2 \pi}}+\sqrt{\frac{\pi}{\alpha}} \frac{\int P_{\text {mod }}^{*}(\Delta K, \Phi) d \Delta K}{T_{\text {int }}}\right)
\end{aligned}
$$

$P_{2}(K, \Phi)$ corresponds to the speckle noise spectrum, which comes from the terms in (A1) related with 'slow time' $t$.

With the definition of the one-way antenna aperture in azimuth $\beta_{\phi}=\frac{2 \sqrt{2 \ln 2} L_{\phi}}{r_{0}}$,we can transform

$$
\frac{q^{\prime} \beta_{d}^{\prime} T_{i n t}}{\sqrt{2 \pi}}=\left(\left(T_{i n t} \frac{2 V}{\lambda} \beta_{\phi} \sin \Phi\right)^{2}+\left(\frac{2}{\sqrt{\pi}} T_{i n t} k \cos \theta \sqrt{m_{t t}}\right)^{2}\right)^{1 / 2}
$$

The first and second term of this equation represent the Doppler bandwidth effect due to the platform motion and to the surface scatters motion, respectively. The first term corresponds to $N_{J a c}(\Phi)$ as expressed in (6). So, with respect to Jackson's model, our model now takes into account the effect induced by the scatter velocities (through $m_{t t}$ ) and takes into account an additional term related to the slope and velocity variances (second term in the parenthesis of A37).

Using the definitions of ( $8 b-8 f)$, one can derive the expression (8a) for the model of speckle noise spectrum.

Finally, the total normalized fluctuation spectrum $P(K, \Phi)$ is $P(K, \Phi)=P_{I R}(K, \Phi) P_{\text {mod }}(K, \Phi)+P_{S P}(K, \Phi)$ where $P_{I R}(K, \Phi)$ is the Fourier Transform of the impulse response function, $P_{I R}(K, \Phi)=\left[\operatorname{tri}\left(\frac{K}{2 \pi K_{p}}\right)\right]^{2}, P_{s p}(K, \Phi)$ is the speckle noise spectrum, $P_{s p}(K, \Phi)=P_{2}(K, \Phi)$.

In the above derivation, the antenna rotation is ignored. If considering the rotation, then $G(\vec{x}-\vec{V} t)$ in (A1) becomes $G\left(\vec{x}-\vec{V}_{s} t\right)$, in which $\vec{V}_{s}$ is the sum of $\vec{V}$ and antenna rotation velocity vector projected on the horizontal surface, our derivation shows this modification does not alter the final results.

\section{APPENDIX B}

Table B: List of variables and functions

\begin{tabular}{|c|l|c|l|}
\hline \multicolumn{2}{|l|}{ Variable List } & $\hat{\alpha}$ & factor proportional to $m_{t t}$, defined in $(8 \mathrm{~g})$ \\
\hline ARE & Average Relative Error & $\beta_{\phi}$ & one-way antenna aperture in azimuth \\
\hline$B$ & bandwidth of the transmit pulse & $\Delta k$ & $\Delta k=k-k^{\prime}$ \\
\hline $\mathrm{C}$ & light speed & $\delta x$ & $\begin{array}{l}\text { horizontal resolution (range resolution projected on } \\
\text { the surface) }\end{array}$ \\
\hline$\Delta K$ & $2 \Delta k \sin \theta$ & $k, k^{\prime}$ & wavenumber of the electromagnetic wave \\
\hline Hs & significant wave height &
\end{tabular}




\begin{tabular}{|c|c|c|c|}
\hline$\kappa$ & the wave number difference of the transmitted pulse & $K$ & the wavenumber at the surface \\
\hline$\vec{K}$ & $2 \kappa \sin \theta \vec{\rho}$ & $K_{i}$ & wave number $(i=1, \ldots, 64)$ \\
\hline$K_{p}$ & inverse of $\delta x$ & $K_{\text {detc_min }}$ & minimal detectable wave number of KuROS \\
\hline$K_{\text {detc_max }}$ & maximal detectable wave number of KuROS & $\lambda$ & wavelength of the electromagnetic wave \\
\hline$\lambda_{p}$ & wavelength at the peak of the wave spectrum & $L_{\phi}$ & azimuthal width of the radar footprint \\
\hline$m s s_{e}$ & effective slope variance of the surface & $m_{t t}$ & surface vertical velocity variance \\
\hline$N$ & $\begin{array}{l}\text { number of averaged spectra in the "post-integration" } \\
\text { method }\end{array}$ & $N_{\text {int }}$ & $\begin{array}{l}\text { number of independent samples due to the ocean } \\
\text { condition }\end{array}$ \\
\hline$N_{J a c}$ & $\begin{array}{l}\text { number of independent samples due to the radar } \\
\text { displacement }\end{array}$ & $N_{m o v}$ & $\begin{array}{l}\text { number of independent samples due to the motion } \\
\text { of radar and the surface }\end{array}$ \\
\hline$N_{\text {platf }}$ & $\begin{array}{l}\text { number of independent samples due to the radar } \\
\text { displacement }\end{array}$ & $N_{\text {surf }}$ & $\begin{array}{l}\text { number of independent samples due to the motion } \\
\text { of the surface }\end{array}$ \\
\hline$N_{t o t}$ & total number of independent samples & $\omega$ & $\begin{array}{l}\text { angular frequency difference of the electromagnetic } \\
\text { wave } \omega=\kappa c\end{array}$ \\
\hline$\omega_{d}$ & $\begin{array}{l}\text { angular frequency limit of the sea waves which } \\
\text { makes the quasi-specular scattering approximation } \\
\text { valid }\end{array}$ & $\Omega$ & inverse wave age \\
\hline$\Omega_{0}$ & Doppler frequency & $\vec{\phi}$ & $\begin{array}{l}\text { unit vector perpendicular to } \vec{\rho} \text { in the horizontal } \\
\text { plane }\end{array}$ \\
\hline$\Phi$ & azimuth angle relative to the flight direction & $\Phi_{i}$ & azimuth direction $(i=1, \ldots, 60)$ \\
\hline$\Phi_{1}$ & azimuth angle with respect to geographical North & PRF & radar pulse repetition frequency \\
\hline$R_{e}$ & effective Fresnel reflectivity & $\vec{\rho}$ & unit vector along the horizontal look direction \\
\hline$R_{\text {int }}$ & $\begin{array}{l}\text { ratio of the contribution of } N_{\text {int }} \text { to the total speckle } \\
\text { noise spectrum }\end{array}$ & SNR & Signal-To-Noise ratio \\
\hline$T_{\text {int }}$ & integration time of the radar echo & $\theta$ & incidence angle \\
\hline$\vec{u}, \vec{u}^{\prime}$ & $\begin{array}{l}\text { two vectors in the horizontal plane defined in (A6) } \\
\text { and (A19), respectively }\end{array}$ & $U_{10}$ & wind speed at $10 \mathrm{~m}$ height \\
\hline$\vec{v}, \vec{v}^{\prime}$ & $\begin{array}{l}\text { two vectors in the horizontal plane defined in (A6) } \\
\text { and (A19), respectively }\end{array}$ & $v, v^{\prime}$ & $\begin{array}{l}\text { angular frequency of the electromagnetic wave } \\
v=k c, v^{\prime}=k^{\prime} c\end{array}$ \\
\hline$V$ & platform speed & $\overrightarrow{x_{l}}$ & $\begin{array}{l}\text { position vector of the ith backscatter sample } \\
\text { projected on the horizon plane }(i=1,2,3,4)\end{array}$ \\
\hline$\vec{w}, \vec{w}^{\prime}$ & $\begin{array}{l}\text { Two distance vectors in the horizontal plane defined } \\
\text { in (A6) and (A19), respectively }\end{array}$ & $\xi_{i}$ & $\begin{array}{l}\text { height of the sea surface at the time of } t \text { at positions } \\
i(i=1,2,3,4)\end{array}$ \\
\hline $\begin{array}{c}\xi_{i, \Delta t} \\
i=1,2,3,4\end{array}$ & the height of the sea surface at the time of $t+\Delta t$ & $\nabla$ & Hamilton operator, $\nabla=\vec{\rho} \frac{\partial}{\partial x_{1}}+\vec{\phi} \frac{\partial}{\partial x_{2}}$ \\
\hline \multicolumn{4}{|l|}{ Function List } \\
\hline$\delta \sigma^{0}(\mathrm{x}, \Phi)$ & $\begin{array}{l}\text { fluctuation function of the measured } \sigma^{0} \text { along the } \\
\text { horizontal axis in the azimuth direction }\end{array}$ & $E_{0}(v)$ & $\begin{array}{l}\text { Fourier Transform of the incident short-pulsed } \\
\text { waveform }\end{array}$ \\
\hline$G(\vec{x})$ & $\begin{array}{l}\text { antenna gain pattern projected on the horizon plane } \\
\text { defined in (A5) }\end{array}$ & $M\left(v, v^{\prime}, \omega, \Delta t\right)$ & $\begin{array}{l}\text { four-frequency moment defined from the surface } \\
\text { scattering transfer function defined in (4) }\end{array}$ \\
\hline$N\left(v, v^{\prime}, \omega, \Omega_{0}\right)$ & $\begin{array}{l}\text { Fourier Transform of the four-frequency moment } \\
\text { defined in (3) }\end{array}$ & $N_{\text {tot_meas }}$ & $\begin{array}{l}\text { empirical estimate of the total number of } \\
\text { independent samples inversed from each azimuthal } \\
\text { estimate of } P_{s p \_m e a s}\end{array}$ \\
\hline$\widetilde{\mathrm{P}}(\omega)$ & $\begin{array}{l}\text { ensemble average fluctuation spectrum of the signal } \\
\text { defined in (1) }\end{array}$ & $\varphi$ & phase defined in (A3a) \\
\hline$P_{\text {mod }}$ & $\begin{array}{l}\text { spectrum of the relative fluctuations due to the } \\
\text { presence of long waves }\end{array}$ & $P_{\text {mod }}^{*}$ & $\begin{array}{l}\text { sum of the modulation spectrum } P_{\bmod } \text { and a second } \\
\text { term related to } \mathrm{m}_{t t} \text { defined in }(8 \mathrm{~h})\end{array}$ \\
\hline$P_{\sigma^{0}}(\mathrm{~K}, \Phi)$ & $\begin{array}{l}\text { spectral density of } \delta \sigma^{0}(\mathrm{x}, \Phi) \text { as a function of the } \\
\text { wave number defined in }(16)\end{array}$ & $\left(P_{\sigma^{0}}\right)_{N T \text { Tint }}$ & $\begin{array}{l}\text { spectrum of signal fluctuation calculated over the } \\
\text { period of } N \cdot T_{\text {int }}\end{array}$ \\
\hline$\left\langle\left(P_{\sigma^{0}}\right)_{\text {Tint }}\right\rangle_{N}$ & $\begin{array}{l}\text { average of } N \text { spectra each calculated over the period } \\
\text { of } T_{\text {int }}\end{array}$ & $\mathrm{P}_{s p}$ & speckle noise spectrum of our model defined in $(8 a)$ \\
\hline $\mathrm{P}_{s p \_J a c}$ & $\begin{array}{l}\text { speckle noise spectrum of Jackson's model defined } \\
\text { in (5a) }\end{array}$ & $P_{S p \_m e a s}$ & $\begin{array}{l}\text { empirical estimates of the omni-directional speckle } \\
\text { spectrum obtained from the KuROS data }\end{array}$ \\
\hline$P_{s p \_m o d}$ & $\begin{array}{l}\text { omni-directional speckle noise spectrum from the } \\
\text { model }\end{array}$ & $S(v, t)$ & surface scattering transfer function defined in (A1) \\
\hline$\sigma_{Q S}^{0}(\theta)$ & $\begin{array}{l}\text { backscattering coefficients approximated by the } \\
\text { Quasi-specular scattering model defined in (9) }\end{array}$ & tri & triangle function \\
\hline$W\left(\Omega_{0}\right)$ & filter window defined in (2) & $\xi(\vec{x}, t)$ & instantaneous elevation of the surface \\
\hline$\Xi(\vec{w}, \Delta t ; \vec{K})$ & defined in (A12) & & \\
\hline
\end{tabular}

\section{REFERENCE}

[1] F.C. Jackson, "An analysis of short pulse and dual frequency radar techniques for measuring ocean wave spectra from satellites," Radio Sci, $16,1385-1400,1981$

[2] G. Engen and H. Johnsen, "SAR-ocean wave inversion using image cross spectra," IEEE Trans. Geosci. Remote Sensing, vol. 33, pp. 1047-1056, 1995.

[3] D. Hauser, G. Caudal, G. J. Rijckenberg, D. Vidal-Madjar, G. Laurent, and P. Lancelin, "RESSAC: a new airborne FM/CW radar ocean wave spectrometer," IEEE Transactions on Geoscience \& Remote Sensing, vol. 30, pp. 981-995, 1992.

[4] D. Hauser, C. Tourain, L. Hermozo, et al, "New observations from the SWIM radar on board CFOSAT: instrument validation and ocean wave measurement assessment, IEEE Transactions on Geoscience \& Remote Sensing, doi 10.1109/TGRS.2020.2994372, 2020

[5] W. R. Alpers, D.B. Ross, and C.L. Rufenach, "On the detectability of ocean surface waves by real and synthetic aperture radar," J. Geophys Res., 86(C7):6481-6498, 1981.

[6] R.C. Beal, D.G. Tilley, and F.M. Monaldo, "Large-and small-scale spatial evolution of digitally processed ocean wave spectra from SEASAT 
synthetic aperture radar", J. Geophys. Res., 88(C3):1761-1778, 1983.

[7] K. Hasselmann and S. Hasselmann, "On the nonlinear mapping of an ocean wave spectrum into a synthetic aperture radar image spectrum and its inversion" J. Geophys. Res., 96, (C6)10713-10729, 1991.

[8] P. W. Vachon, H.E. Krogstad, and J.S. Paterson, "Airborne and spaceborne synthetic aperture radar observations of ocean waves," $J$. Atmosphere, 32(1):83-112, 1994.

[9] D. Hauser, C. Tison, T. Amiot et al, "SWIM: The First Spaceborne Wave Scatterometer," IEEE Transactions on Geoscience and Remote Sensing, 55(5):3000-3014, 2017.

[10] G. Caudal, D. Hauser, R. Valentin, et al, "KuROS: A New Airborne KuBand Doppler Radar for Observation of Surfaces," Journal of Atmospheric and Oceanic Technology, 31(10):2223-2245, 2014.

[11] F.C. Jackson, W.T. Walton, and P.L. Baker, "Aircraft and satellite measurement of ocean wave directional spectra using scanning-beam microwave radars," J. Geophys. Res., 90(C1):987, 1985.

[12] E. Le Merle, D. Hauser, and C. Tison, "Directional wave spectra at the regional scale with the KuROS airborne radar: comparisons with models,' Ocean Dynamics, 69(6):679-699, doi 10.1007/s10236-019-01271-5, 2019.

[13] O. Boisot, L. Amarouche, J.-C. Lalaurie, et al., "Dynamical Properties of Sea Surface Microwave Backscatter at Low-Incidence: Correlation Time and Doppler Shift," IEEE Transactions on Geoscience and Remote Sensing, 54(12):7385-7395, 2016

[14] F.T. Ulaby, R.K. Moore and A.K. Fung, "Radar Remote Sensing and Surface Scattering and Emission Theory," Microwave Remote Sensing, Vol. II, Artech House Publisher, 1982.

[15] M.S. Longuet-Higgins, "The effect of nonlinearities on statistical distributions in the theory of sea waves," J. Fluid Mech, 17, 459-480, 1963

[16] D.R. Thompson, T. M.Elfouhaily, and J. L. Garrison, "An improved geometrical optics model for bistatic GPS scattering from the ocean surface," IEEE Transactions on Geoence \& Remote Sensing, 43(12):2810-2821, 2005.

[17] T. Elfouhaily, B. Chapron and K. Katsaros, "A Unified Directional Spectrum for Long and Short Wind-Driven Waves," J. Geophys. Res., Vol. 102, No. C7, pp. 15781-15796, 1997.

[18] S.L. Durden, and J.F. Vesecky, "A physical radar cross-section model for a wind-driven sea with swell," IEEE Journal of Oceanic Engineering, 10(4):445-451, 1985.

[19] T. Elfouhaily, and C.A. Guérin, "A critical survey of approximate scattering wave theories from random rough surfaces, Waves in Random Media",14(4), 1 -40, 2004.

[20] A. Mouche, B. Chapron, and N. Reul, "Importance of the sea surface curvature to interpret the normalized radar cross-section," J. Geophys. Res., 112(C10), 2007.

[21] F. Ardhuin, P. Brandt, L. Gaultier et al, "SKIM, a candidate satellite mission exploring global ocean currents and waves," Front. Mar. Sci., doi 10.3389/fmars.2019.00209, 2019

[22] P. Beckmann, and A. Spizzichino, "The Scattering of Electromagnetic Waves from Rough Surfaces", Pergamon publisher, pp.503,1963.

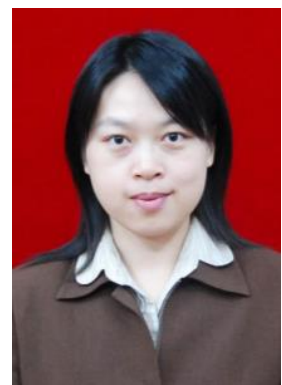

Ping Chen received the M. E. degree in computing science from Wuhan University, Wuhan, China, in 1998, and the $\mathrm{Ph}$. D degree in information and communications engineering from Huazhong University of Science and Technology, Wuhan, China, in 2002. She is current a professor with the School of Electronic Information and Communications, Huazhong University of Science and Technology. Her research interest lies in electromagnetic wave scattering theory and ocean wave spectrum detection by wave scatterometer. From 2014-2015, she was a Visiting Researcher with Laboratoire Atmosphres, Milieux, Observations Spatiales, France, where she worked on wave parameter retrieval with doppler radar.

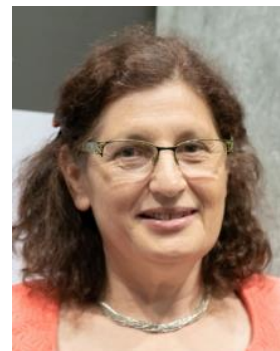

Danièle Hauser is Senior Scientist at CNRS (Centre National de la Recherche Scientifique) and develops her research activity at LATMOS (Laboratoire Atmosphère, Milieux, Observations Spatiales). She received a $\mathrm{PhD}$ Thesis in meteorology in 1980 and a State Thesis in Physics in 1989. She is working for more than 25 years on microwave observations of the ocean surface (surface wind, waves, salinity) and air/sea interactions studies. She is presently Principal Investigator of the CFOSAT mission dedicated to the global measurement of wind and waves from satellite.

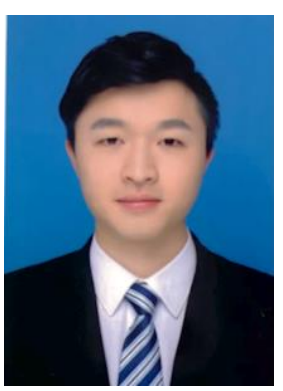

Shihao Zou received the M.S. degree in electromagnetic field and microwave technology from the School of Electronic Information and Communications, Huazhong University of Science and Technology, Wuhan, China, in 2020. He worked on the research of nonlinear random sea and the modification of speckle spectrum theory between 2017 and 2020 .

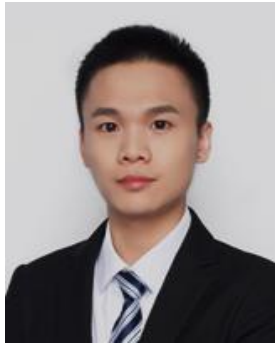

Jianyang Si is current a postgraduate student in electromagnetic field and microwave technology from the School of Electronic Information and Communications, Huazhong University of Science and Technology, Wuhan, China. He is occupied in the wave spectrum detection by wave scatterometer and his research interest consists in the improvement of wave spectrum inverse algorithm.

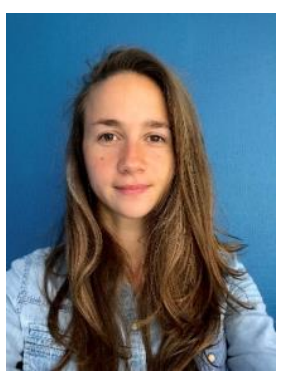

Eva Le Merle received the Master degree in physics for remote sensing from Université Denis Diderot, Paris, France in 2016. She received the $\mathrm{Ph} . \mathrm{D}$. degree in oceanography and remote sensing from Université Paris-Saclay, France in 2019. Her thesis was dedicated to the study of the physical properties of ocean wave using the airborne KuROS radar data. She is currently post-doc at LATMOS (CNRS) in France where she continues to study the physical properties of ocean wave using the CFOSAT data. 